\title{
Hybrid approach to modeling spatial dynamics of systems with generalist predators
}

\author{
Elizabeth F. Fussell, Andrew L. Krause, and Robert A. Van Gorder* \\ Mathematical Institute, University of Oxford, Andrew Wiles Building, Radcliffe Observatory Quarter, Woodstock Road, Oxford, OX2 6GG, UK \\ *Robert.VanGorder@maths.ox.ac.uk
}

\begin{abstract}
We consider hybrid spatial modeling approaches for ecological systems with a generalist predator utilizing a prey and either a second prey or an allochthonous resource. While spatial dispersion of populations is often modeled via stepping-stone (discrete spatial patches) or continuum (one connected spatial domain) formulations, we shall be interested in hybrid approaches which we use to reduce the dimension of certain components of the spatial domain, obtaining either a continuum model of varying spatial dimensions, or a mixed stepping-stone-continuum model. This approach results in models consisting of partial differential equations for some of the species which are coupled via reactive boundary conditions to lower dimensional partial differential equations or ordinary differential equations for the other species. In order to demonstrate the use of this approach, we consider two case studies. In the first case study, we consider a one-predator two-prey interaction between beavers, wolves and white-tailed deer in Voyageurs National Park. In the second case study, we consider predator-prey-allochthonous resource interactions between bears, berries and salmon on Kodiak Island. For each case study, we compare the results from the hybrid modeling approach with corresponding stepping-stone and continuum model results, highlighting benefits and limitations of the method. In some cases, we find that the hybrid modeling approach allows for solutions which are easier to simulate (akin to stepping-stone models) while maintaining seemingly more realistic spatial dynamics (akin to full continuum models).
\end{abstract}

Keywords: spatial ecology, predator-prey interactions, spatial dynamics, generalist predator

\section{Introduction}

Mathematical modeling of interactions between coexisting species is an important tool in ecology, with a wide range of practical applications. In particular, understanding ecosystem dynamics can help to streamline conservation efforts, for example by uncovering the impact of human activity in marine ecosystems (Crowder and Norse, 2008) or predicting the spread of an invasive species (Okubo et al., 1989; Williamson, 1996; Murray, 2002). Mathematical models show that the spatial complexity of an ecosystem, and the way in which species move and interact can have a fundamental impact on population dynamics (Holmes et al., 1994; Tilman and Kareiva, 1997).

Many population models assume that all of the interacting species exist at the same point (or are well-mixed such that spatial dispersal can be neglected) and can thus be modeled by a system of ordinary differential equations (ODEs). This assumption dates back to the origins of population dynamics, when Malthus discussed the growth of populations with 'ample resources' which could grow exponentially (Malthus and Appleman, 1976). This was later extended by Verhulst to incorporate resource limitations (Bacaër, 2011) in the form of the Logistic Equation. The classical predator-prey model proposed independently by Lotka and Volterra (Volterra, 1928) was based on the principle of mass action, and assumed that the rate of predation is proportional to the densities of the predator and prey. The Lotka-Volterra equations neglect environmental limitations and predict unbounded growth of the prey in the absence of predation. A more realistic model for many species accounts for the fact that, in the absence of predation, the prey population is more accurately governed by Verhulst's Logistic Equation. Holling (1959) extended this theory to include satiation, whereby the predator is only able to consume a limited amount of prey in a finite amount of time:

$$
\frac{d x}{d t}=r x\left(1-\frac{x}{k}\right)-\theta\left(\frac{x}{x+h}\right) y, \quad \frac{d y}{d t}=\theta\left(\frac{\epsilon x}{x+h}\right) y-\delta y .
$$

Here $x$ and $y$ are the biomass of the prey and predator populations, $r$ is the intrinsic growth rate of the prey; $k$ is the prey's carrying capacity, which determines how readily the environment can sustain the prey; $\theta$ is the maximum rate at which predators can consume prey; $h$ is a half-saturation constant; $\epsilon$ is the conversion efficiency of prey into new predators; and $\delta$ is the predator death rate. This model is typically referred to as the Rosenzweig-Macarthur model, due to a seminal paper on its analysis and applications to predator-prey interactions (Rosenzweig and MacArthur, 1963).

ODE models such as (1) provide a good basis for modeling species interactions. They are simple and hence accessible to a variety of different scientists, can be easily adapted to account for environmental factors, and produce analytically tractable results. For these reasons they have traditionally been favored by the biological community. However, the influence of spatial effects, in particular motion or diffusion of populations, can often play a role in modifying such ODE dynamics (Levin, 1974, 1976; Scheffer and De Boer, 1995; Weisser and Hassell, 1996; 
Weisser et al., 1997; Jansen, 1995; Ripa, 2000; Jansen et al., 2000; Jansen, 2001; Okubo and Levin, 2013; Pilyugin et al., 2016). In some cases, spatial dispersion of predator or prey (particularly when this dispersion results in spatial heterogeneity within the population distributions) may have a stabilizing effect on the dynamics of the model (Jansen, 1995; Gurney and Veitch, 2000; Briggs and Hoopes, 2004; Goldwyn and Hastings, 2008, 2009; Ruokolainen et al., 2011). Spatial dispersal can also lead to more complex spatiotemporal behavior, possibly accounting for observed variation in species' abundance, or enhancing persistence across large regions (Hassell et al., 1991; Ranta et al., 1997; Liebhold et al., 2004; Petrovskii et al., 2004).

Two common paradigms for modeling spatial variation in predator-prey systems are stepping-stone models (or, network models) which formulate space as a collection of discrete points or locations, and continuum models, which formulate dynamics on explicit spatial domains. Nonlocal dispersal, structured populations, and other complicated effects can be captured by more complex mathematical frameworks, such as integrodifferential or integrodifference equations (Andersen, 1991; Van Kirk and Lewis, 1997). For simplicity, we will focus on stepping-stone and spatially continuous models which involve classical differential equations, and which are widely used to capture spatial variation in population dynamics.

Stepping-stone models assume that space can be partitioned into discrete groups or islands, and these are modeled mathematically by way of networks (Kareiva, 1990; Neubert et al., 2002; Amarasekare, 2007; Baggio et al., 2011). Steppingstone models have seen applications in the conservation of species living in fragmented habitats (Burkey, 1989; Lima, 2002) as well as in population genetics (Shiga, 1988). Recent works have considered generalist predators on steppingstone domains, with the abundance of each food source varying at each patch in the network (Levy et al., 2016; Shen and Van Gorder, 2017). In Jansen (1995); Jansen and Lloyd (2000); Neubert et al. (2002); Plitzko and Drossel (2015); Shen and Van Gorder (2017), it was seen that the stepping-stone model may modify non-equilibrium dynamics such as limit cycles in generalist predator dynamics. Non-equilibrium dynamics, such as limit cycles, destabilize the predator and prey populations, and can occur in non-intuitive settings such as in the paradox of enrichment (Roy and Chattopadhyay, 2007). Such destabilization (or lack thereof) can have important consequences for conservation and persistence of coexisting species (Rosenzweig, 1971). A two-patch system with a migratory predator moving between patches with separate prey-population on each patch was found to be stabilized by dispersion of the predator (Holt, 1984), whereas a corresponding model with two prey populations and one predator in a single patch was been shown to exhibit a variety of non-equilibrium dynamics (Hutson and Vickers, 1983). On the other hand, Jansen (2001) considered a two-patch Rosenzweig-MacArthur model with a predator and prey population on each patch, where the predator was allowed to migrate by discrete diffusion, finding quasi-periodic and chaotic oscillations. Chaotic dynamics and multistability were also observed by Suzuki and Yoshida (2012) when studying a three-trophic-level (predator-prey-resource) system con- nected by dispersal. Shen and Van Gorder (2017) considered a variety of network configurations of greater than three nodes, and found that increasing the predator migration rate may stabilize or destabilize the dynamics, depending on the specific network configuration employed.

Other models consider space as a continuum, and motion as diffusion (or, Brownian motion) over this continuous domain. This results in partial differential equations (PDEs) for all species which both move in time and in space (Levin, 1976; Kareiva, 1990; Cantrell and Cosner, 2004; Bassett et al., 2017). One advantage of using continuum models is the ability to observe spatial patterning on scales determined by both nonlinear species' interactions as well movement rates. Interactions which are stable in the absence of diffusion can give rise to spatially heterogeneous solutions when diffusion is added (Alonso et al., 2002; Murray, 2002; Baurmann et al., 2007). Additionally, environmental features and structure can be considered with an arbitrary precision, allowing gradients or localization of resources and hazards (Holmes et al., 1994).

In the case of a generalist predator, Bassett et al. (2017) extended the ODE model of Nevai and Van Gorder (2012) to a continuous spatial domain with spatially heterogeneous allochthonous resource distributions, and patterning induced by a spatially localized allochthonous resource was possible. Furthermore, dynamics from PDE predator-prey systems can still admit oscillatory or even chaotic solutions in some regimes (Pascual, 1993). Indeed, the emergence of spatio-temporal chaos, permitting spatial regions of relatively large populations which offset regions of negligibly small populations, has been suggested as one resolution to the paradox of enrichment (Petrovskii et al., 2004).

At first glance, continuum models would seem like an obvious choice for modeling two-dimensional spatially continuous habitats. However, this approach has several important limitations. Mean-field approximations of populations (and other approaches for justifying classical differential equation-type models) require a suitably large number of interacting individuals, especially if one is considering their density over space to evolve according to a PDE (Metz, 2000). In this sense, considering a well-mixed but sparse population to evolve in time but neglecting space may be more appropriate than modelling detailed spatial evolution which may no longer correspond to the population of interest. Additionally, in comparison with the more analytically tractable ODE models, the continuum models less readily permit the consideration of environmental stochasticity (Crowley, 1981), and involve more parameters which need to be estimated. Moreover, there exist habitats such as networks of islands surrounded by sea, which would not naturally fit into the continuum framework. Understanding the impact of complex environments on population interactions is an important area of research, and models with few tunable parameters which can still capture spatial variability are invaluable in understanding interacting and dispersing populations (Hanski, 1998). Additionally, a system of PDEs is less computationally tractable than a system of ODEs, so it would be useful to know for which cases a high level of spatial detail adds value and for which cases simpler models are sufficient to recapitulate the 
dynamics of interacting populations.

Density-dependent dispersion (Huang and Diekmann, 2001; Nathan, 2001; Hauzy et al., 2007; El Abdllaoui et al., 2007) has also been considered in stepping stone models (Hauzy et al., 2010; Kang et al., 2015) as well as continuum models (Holmes et al., 1994). In such models, not only will the spatial position matter, but the concentration of a species at that location in space will also modify diffusion of the species. Additionally, there exist models which include conditional dispersion, or advection toward resource or fecundity gradients, rather than simple Brownian motion (Grindrod, 1988; Armsworth and Roughgarden, 2005; Chen et al., 2008; Kurowski et al., 2017). Spatial structure, both of the domain and the constituent populations, is essential to the dynamics emergent from such models; indeed, such models have no sensible ODE analogue, and hence one must consider spatial effects in more detail.

Spatially-homogeneous ODE, spatially discrete steppingstone, and spatially continuous PDE models can provide valuable insights into species interactions, but making a rigid choice between the three approaches is limiting. Many ecological situations can be best understood by combining features of multiple modeling approaches. For example, we see features of both stepping-stone and continuum models in a system where a predator disperses across a large range in search of distinct prey colonies, with minimal prey migration between colonies. In other scenarios, we may only be interested in observing spatial effects in one part of the domain with high fidelity, and neglecting detailed spatial effects in other regions (either for simplicity or due to lack of information necessary for accurate parameter estimation). In these cases, a hybrid continuum-stepping-stone model may be more appropriate. Such a hybrid model could employ PDEs in some parts of the domain and lower dimensional PDEs or ODEs on subregions of the domain, such as boundaries. For example, one could couple a two-dimensional PDE with one-dimensional PDEs on the boundaries of the domain, or couple a one-dimensional PDE with ODEs. These kinds of models have seen applications in diverse fields ranging from the modeling of hydrocarbon production in rock fractures (Moinfar et al., 2013; Council, 1996) to the modeling of tumour growth in mathematical physiology (Anderson, 2005). The reduction to lower-dimensional models, say an ODE from a PDE, has previously been considered in ecology, but often the dimension of the entire system is reduced (Maciel and Kraenkel, 2014), while we shall consider situations where only part of the spatial domain is reduced, resulting in a mixed domain with spatial components of differing dimension.

In the present paper we shall focus on spatial modeling approaches for ecological systems with a generalist predator which utilizes a prey and then either a second prey or an allochthonous resource. In order to extend (1) to a generalist predator, note that Nevai and Van Gorder (2012) considers the case where a generalist predator feeds on both prey and a secondary food source in the form of an allochthonous resource. On the other hand, Yamauchi and Yamamura (2005) propose a model where the generalist predator has two prey, which both undergo logistic growth. We shall consider the extension of both of these ecological systems under a hybrid spatial model- ing approach.

In order to better understand these spatial modeling paradigms and to develop a hybrid modeling approach, we shall first formulate prototypical models in Section 2. In Section 3 we shall introduce our hybrid modelling approach, and relate it to the other modelling frameworks discussed. We shall then compare these modeling approaches through two case studies. In Section 4, we consider different spatial models for a one-predator two-prey interaction between wolves, deer, and beavers. In Section 5, we consider predator-prey-allochthonous resource interactions between bears, salmon, and berries. For each case study, we will make comparisons between the four models and weigh the benefits and costs of using each approach. We discuss our results in Section 5.

\section{Canonical modeling approaches}

We first present a description of three canonical modeling approaches of a three species interaction between a predator $y$, prey $x$, and the predator's secondary food source $s$ (which may be either an allochthonous resource, or a second prey) involving continuous time mathematical models for population densities. In the first case we consider ODE models which neglect all spatial effects, and in the other extreme we consider continuum models, which aim to make no simplification of the spatial domain. We will also discuss a common approach to discretizing space into stepping-stone domains. In the following sections, we investigate the similarities and differences between the solutions of the four models under ecologically relevant case studies.

We remark that the nonlinear interaction terms in these equations will depend implicitly on the spatial scale, even when dispersal is not accounted for. In the next subsections we will consider different models of spatial dispersal of populations but will assume the same population interactions will occur locally in space, which is a simplification for two reasons. Firstly, parameters in models over different spatial scales will have different dimensional properties, as the population density will be with respect to the space within which the populations are evolving, and hence will vary in their numerical values. More striking, however, is that it is not clear a priori that the same functional responses should necessarily carry across scales, as the individual and probabilistic interactions between organisms may emerge differently at different scales, leading to a variety of possibly nonequivalent models (Kareiva, 1990; Tilman and Kareiva, 1997; ter Braak et al., 1998; Levin, 2003; Guo et al., 2005). Here we will assume the same functional responses in species' interactions for simplicity, as is common, and assume parameters that are of the same order of magnitude, which we vary in order to elucidate qualitative differences between these approaches. We do not claim to suggest quantitative differences which may be due to details specific to a particular ecological setting, but instead we will emphasize qualitative phenomena such as population persistence, oscillations, and spatial organisation. 


\subsection{ODE models}

ODE models are the mathematically simplest way of modeling populations evolving in continuous time, where any spatial dependence of the population is neglected, so that only the average or total population dynamics are modeled. The domain is treated as a single point in space and the system is governed by three ordinary differential equations:

$$
\begin{aligned}
& \frac{d x}{d t}=f(x)-g(x, s, y), \\
& \frac{d s}{d t}=F(s)-G(x, s, y), \\
& \frac{d y}{d t}=H(x, s, y)-\delta y,
\end{aligned}
$$

with initial conditions $x(0)=x_{0}, s(0)=s_{0}, y(0)=y_{0} \in[0, \infty)$. The functions $f(x)$ and $F(s)$ account for the growth and intrinsic mortality (intraspecies competition) of the prey and secondary food source in the absence of predators. The growth term could take a variety of forms, including exponential, logistic, parabolic, or Gompertz forms, and should be selected based on prior knowledge of the species and environmental conditions (Gamito, 1998). The functions $g(x, s, y)$ and $G(x, s, y)$ represent the effect of predation on the prey and the secondary food source, and any cross-dependence here is due to effects of the total food resources on predation (e.g. due to satiation). The function $H(x, s, y)$ is often a linear combination of $g(x, s, y)$ and $G(x, s, y)$ and represents the benefit to the predator gained by consuming prey and secondary food source. Finally, $\delta$ is the predator mortality rate.

There are several possible formulations of this generalist predator model, but for concreteness we will use the basic Rosenzweig-Macarthur model given by (1) with a specific secondary predator resource. We investigate two possible models of a secondary predator resource: an allochthonous subsidy, and a secondary prey species. Nevai and Van Gorder (2012) proposed a further extension of (1), where the predator, $y$, feeds on both prey, $x$, and a secondary food source in the form of an allochthonous resource, $s$ :

$$
\begin{gathered}
\frac{d x}{d t}=r x\left(1-\frac{x}{k}\right)-\theta\left(\frac{x}{x+s+h}\right) y, \\
\frac{d s}{d t}=i-\gamma s-\psi\left(\frac{s}{x+s+h}\right) y, \\
\frac{d y}{d t}=\left(\frac{\epsilon \theta x+\eta \psi s}{x+s+h}\right) y-\delta y,
\end{gathered}
$$

where $\psi$ and $\eta$ are the rate and efficiency of the allochthonous resource or subsidy consumption respectively, $i$ is the allochthonous resource or subsidy input rate and $\gamma$ is the natural decay rate of the allochthonous resource.

Instead of an allochthonous resource, the predator's secondary food source could take the form of a secondary prey, as in Yamauchi and Yamamura (2005), where the predator, $z$, has two prey, $x$ and $y$, which both undergo logistic growth. In this case, the governing equations are:

$$
\begin{gathered}
\frac{d x}{d t}=r_{1} x\left(1-\frac{x}{k}\right)-\theta\left(\frac{x}{x+y+h}\right) z, \\
\frac{d y}{d t}=r_{2} y\left(1-\frac{y}{K}\right)-\psi\left(\frac{y}{x+y+h}\right) z, \\
\frac{d z}{d t}=\left(\frac{\epsilon \theta x+\eta \psi y}{x+y+h}\right) z-\delta z .
\end{gathered}
$$

The functional response of the predator in these models is the natural extension of the Holling Type II functional response seen in (1). It accounts for the fact that the predator can now be satiated by both food sources.

We note that in both of the above models, the satiation term involves a sum of either the density of the prey and the subsidy, or the density of two distinct kinds of prey, and these are not generally commensurate quantities. The studies where we have borrowed these nonlinear interaction terms from, (Nevai and Van Gorder, 2012; Yamauchi and Yamamura, 2005), get around this in two distinct ways. The first considers the models to be over total biomass entering and leaving the predatorprey-subsidy compartments, and hence the system is already dimensionally consistent in modelling transfer of biomass between species. The second study instead defines a predator encounter rate and attack probability for each prey, and then multiplies both prey terms by the handling time effectively scaling this sum to be dimensionless. For brevity we will assume such choice of nondimensionalization has already been carried out, noting that dimensional consistency is an important modeling requirement.

\subsection{Stepping-stone models}

We now consider discrete spatial domains consisting of $N$ nodes or patches, representing distinct spatial regions. Within each patch spatial detail is neglected, as the populations are assumed to be well-mixed with no net dispersal within a patch. Assuming that the predator, prey, and secondary food source are each defined at all $N$ nodes, we have a system of $3 N$ ordinary differential equations:

$$
\begin{aligned}
& \frac{d x^{(j)}}{d t}=f^{(j)}\left(x^{(j)}\right)-g^{(j)}\left(x^{(j)}, s^{(j)}, y^{(j)}\right)+\sum_{i=1}^{N}\left(A_{i, j} x^{(i)}-A_{j, i} x^{(j)}\right), \\
& \frac{d s^{(j)}}{d t}=F^{(j)}\left(s^{(j)}\right)-G^{(j)}\left(x^{(j)}, s^{(j)}, y^{(j)}\right)+\sum_{i=1}^{N}\left(B_{i, j} y^{(i)}-B_{j, i} y^{(j)}\right), \\
& \frac{d y^{(j)}}{d t}=H\left(x^{(j)}, s^{(j)}, y^{(j)}\right)-\delta^{(j)} y^{(j)}+\sum_{i=1}^{N}\left(C_{i, j} y^{(i)}-C_{j, i} y^{(j)}\right) .
\end{aligned}
$$

For nodes $j=1, \ldots, N$, we define adjacency matrices $A=\left(A_{i, j}\right)$ for the prey, $B=\left(B_{i, j}\right)$ for the allochthonous resource or second prey, and $C=\left(C_{i, j}\right)$ for the predator which specify edges for the passage of prey, secondary food source, and predator between nodes as well as the permissible directions of travel. 
In the present paper we will assume that $A, B$, and $C$ are symmetric, so that the underlying graph is undirected. This ensures that if travel is allowed in one direction it must also be permitted in the opposite direction. We note that the diagonal entries of these matrices do not enter into (11)-(13), so we set $A_{i, i}=B_{i, i}=C_{i, i}=0$ for $i=1, \ldots, N$. If a species does not move, its adjacency matrix can simply be set to a matrix of zeros, leaving interactions between species in disjoint spatial patches being mediated only by mobile species. In the limiting case where all three adjacency matrices are identically zero, then this corresponds to $N$ uncoupled ODE models. We also mention that the dependence of the kinetic functions on the nodal variable $j$ allows for stepping-stone models where some species are not present in some nodes, or other spatial heterogeneity is present. While equations (11)-(13) consist of coupled systems of ODEs, for the sake of nomenclature, we will always refer to this kind of model as a stepping-stone model.

\subsection{Continuum models}

In continuum models, all three species are modeled as dispersing in two spatial dimensions, and the local density of the population is modeled at every point in space. Dispersal is modeled by assuming random migration of individuals leading to diffusive fluxes of each species with diffusion coefficients $d_{1}$, $d_{2}$, and $d_{3}$ representing the rate of movement along density gradients. The relative sizes of these coefficients will depend on the typical range of each species (Lewis et al., 2013; Okubo and Levin, 2013). For example, if the secondary food source is a plant or slow moving prey, we will impose $d_{2} \ll d_{1}, d_{3}$. This system can be written as three partial differential equations:

$$
\begin{gathered}
\frac{\partial x}{\partial t}=d_{1} \Delta x+f(x, \alpha, \beta)-g(x, s, y, \alpha, \beta), \\
\frac{\partial s}{\partial t}=d_{2} \Delta s+F(s, \alpha, \beta)-G(x, s, y, \alpha, \beta), \\
\frac{\partial y}{\partial t}=d_{3} \Delta y+H(x, s, y, \alpha, \beta)-\delta y,
\end{gathered}
$$

where $\Delta=\frac{\partial^{2}}{\partial \alpha^{2}}+\frac{\partial^{2}}{\partial \beta^{2}}$ is the Laplacian operator on the spatial variables $(\alpha, \beta)$. The initial conditions are $x(\alpha, \beta, t=0)=x_{0}(\alpha, \beta)$, $s(\alpha, \beta, t=0)=s_{0}(\alpha, \beta), y(\alpha, \beta, t=0)=y_{0}(\alpha, \beta)$. The prey $x$ is defined on $\Omega_{x}$, the secondary food source $s$ is defined on $\Omega_{s}$, and the predator $y$ is defined on $\Omega_{y}$. We denote with $\Omega$ the whole domain and impose $\Omega=\Omega_{x} \cup \Omega_{s} \cup \Omega_{y}$. The choice of these three domains allows for a variety of models of habitats, depending on their intersections. In some cases, it is useful to think of population interactions only occurring on the boundary between disjoint regions, such as land-based predators consuming fish around the edges of bodies of water, and we will explore such models in detail in the next section. Finally, the spatial heterogeneity of the kinetics (dependence on $\alpha$ and $\beta$ ) will be used here to demarcate regions where species are present, rather than modeling any explicit environmental heterogeneity.

A variety of domains and boundary conditions have been used to model confined populations, sources of invading species, etc. For simplicity, we consider no flux boundary conditions

$$
\begin{array}{ll}
-\mathbf{n} \cdot \nabla x=0, & (\alpha, \beta) \in \partial \Omega_{x}, \\
-\mathbf{n} \cdot \nabla s=0, & (\alpha, \beta) \in \partial \Omega_{s}, \\
-\mathbf{n} \cdot \nabla y=0, & (\alpha, \beta) \in \partial \Omega_{y},
\end{array}
$$

on some parts of the boundary, where $\nabla=\left(\frac{\partial}{\partial \alpha}, \frac{\partial}{\partial \beta}\right)$, $\partial \Omega$ represents the boundary of the region $\Omega$, and $\mathbf{n}$ is the outward unit normal along this boundary. For instance, all external boundaries will use these conditions, so that overall, all populations are confined to $\Omega$. Similarly, internal boundaries where some species are unable to move into another region (such as a body of water) will use these conditions if there is no interaction between these species in neighboring regions.

We will also consider reactive boundary conditions at boundaries of the domain where $\Omega_{y}$ meets $\Omega_{x}$ or $\Omega_{s}$, in order to represent predation in the case that these species only interact along the boundary. For example, one could have a situation where $\Omega_{s}$ and $\Omega_{y}$ share an overlapping region, but $\Omega_{x}$ only meets $\Omega_{y}$ along a line $L=\partial \Omega_{y} \cap \partial \Omega_{x}$. Then $g(x, s, y)$ does not appear in Equation (14), and we write $H(x, s, y)=H_{1}(s, y)$ in $\Omega_{y}$ to represent predation only on $s$, and we instead have the reactive boundary conditions

$$
\begin{array}{ll}
-d_{1} \mathbf{n} \cdot \nabla x=-g(x, y), & (\alpha, \beta) \in L, \\
-d_{3} \mathbf{n} \cdot \nabla y=H_{2}(x, y), \quad & (\alpha, \beta) \in L,
\end{array}
$$

where $H_{2}(x, y)$ is the predation of the predators on the prey $x$ along this boundary. These boundary conditions represent a flux of the species into or out of the domain corresponding to the rate of predation, which depends on the local densities of the populations. We will derive them via a limiting argument in the next section.

\section{General spatial modeling and hybrid models}

Here we will present a method to reduce 'high-dimensional' continuum models into lower dimensional ones, as well as consider hybrid modeling approaches whereby one part of the domain becomes lower-dimensional while retaining an interaction with the higher-dimensional regions of the domain. This will allow us to describe all of the modelling frameworks above as reductions of some 'higher-resolution' spatial model, assuming that such a model is valid. The use and justification of the various ODE and PDE models above can vary widely depending on the ecological system of interest. For instance, if there are too few individuals to justify a continuous population in a small region of space but sufficiently many individuals over a larger region, then it may be unreasonable to represent the population using spatially continuous models, but averaged ODE models may be reasonable approximations. In particular, even if one has suitably detailed information about the environment and dispersal of various organisms in some region, it is not necessarily true that the populations are sufficiently large enough to invoke the continuum hypothesis and consider the population dynamics to evolve in continuous space, time, and density. 
It may instead be the case that these necessary criteria are only satisfied if space is discretized into patches, leading to stepping stone models, or if populations are averaged across the whole region (and dispersal is negligible), island (ODE) models. Nevertheless, here we will begin with spatially continuous models and then reduce these in various limits, assuming that the necessary population sizes and dispersal mechanisms are appropriate for such a model to be valid. One could of course work in the other direction, expanding the dimension of modelling components where necessary to account for populations with different characteristics in sub-regions. In this direction one would want to derive each component via individual-based models and average these suitably to obtain population dynamics at different scales (Metz, 2000; Levin, 2003). We leave such derivations to future work.

\subsection{Example of Dimensional Reduction: Reactive Boundary Conditions}

Consider the setting of the continuum model from Section 2.3. We will use a simple situation to provide a justification for the boundary conditions given by (20)-(21). We consider the density of a predator $y$, such as bears, that primarily forage for food on land, but also wade into the shallow part of a river to hunt salmon, whose density we denote by $x$. The salmon will inhabit both the shallow and deep parts of the river, so we denote the region where both populations overlap as the region $\Omega_{\varepsilon}=\Omega_{x} \cap \Omega_{y}$. An alternative population interaction with similar geometric complexity would be competing bacterial phenotypes within biofilms, for which a continuum model may be more appropriate (Nadell et al., 2016). We parameterize this region by a 'width' $\varepsilon>0$, and in the limit $\varepsilon \rightarrow 0$, we assume that the two-dimensional region collapses smoothly to a boundary curve between the land and the river, i.e. $\lim _{\varepsilon \rightarrow 0} \Omega_{\varepsilon}=L$. A diagram of these regions is given in Figure 1.

Neglecting any other population interactions (such as the second prey or resource $s$ ), and assuming the prey growth kinetics are spatially homogeneous, the population dynamics can be described by

$$
\begin{gathered}
\frac{\partial x}{\partial t}=d_{1} \Delta x+f(x)- \begin{cases}\frac{g(x, y)}{\varepsilon}, & (\alpha, \beta) \in \Omega_{\varepsilon}, \\
0, & (\alpha, \beta) \notin \Omega_{\varepsilon},\end{cases} \\
\frac{\partial y}{\partial t}=d_{3} \Delta y-\delta y+ \begin{cases}\frac{H_{2}(x, y)}{\varepsilon}, & (\alpha, \beta) \in \Omega_{\varepsilon}, \\
0, & (\alpha, \beta) \notin \Omega_{\varepsilon},\end{cases}
\end{gathered}
$$

where the scaling by $\varepsilon$ does not indicate any dimensional relationship but will instead be used to approximately retain the total amount of predation in the limit $\varepsilon \rightarrow 0$. It is typical for $\mathrm{H}_{2}$ and $g$ to be proportional, as in equation (1). On all other boundaries except for internal boundaries along $\partial \Omega_{\varepsilon}$, we assume that these species are confined (e.g. that (17) and (19) hold). We will also assume that the kinetic functions $g$ and $H_{2}$ are sufficiently smooth, and that solutions to (22)-(23) are uniformly convergent in the width of the overlap region, $\varepsilon$, to avoid any technical analytical issues. Similarly, we assume reasonably smooth geometric properties of this limiting process (e.g. that

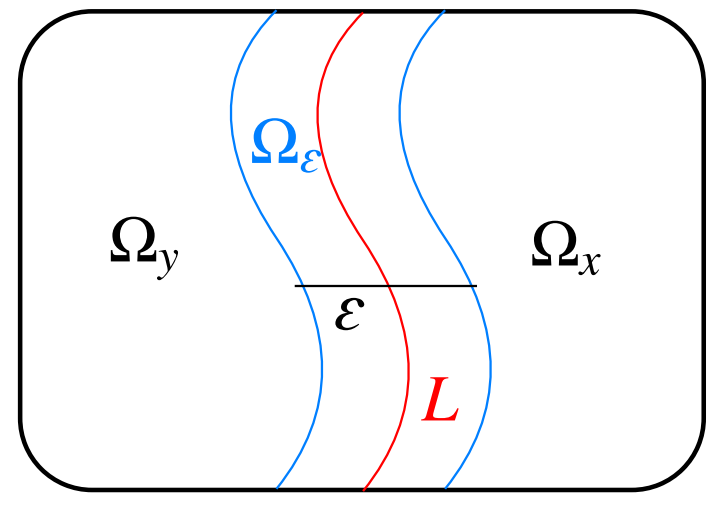

Figure 1: A diagram of the overlapping spatial domain given by (22)-(23). In the limit $\varepsilon \rightarrow 0$, the overlap region $\Omega_{\varepsilon}$ will become the curve $L$.

we are reducing a two dimensional set to a one dimensional smooth curve in a smooth manner).

Integrating (22)-(23) over $(\alpha, \beta) \in \Omega_{\varepsilon}$, and taking the limit $\varepsilon \rightarrow 0$, we see that the time derivatives and spatially homogeneous kinetics will vanish in the limit so that we obtain

$$
d_{1} \lim _{\varepsilon \rightarrow 0} \int_{(\alpha, \beta) \in \partial \Omega_{\varepsilon}} \mathbf{n} \cdot \nabla x d S=\lim _{\varepsilon \rightarrow 0} \int_{(\alpha, \beta) \in \Omega_{\varepsilon}} \frac{g(x, y)}{\varepsilon} d V
$$

and

$$
d_{3} \lim _{\varepsilon \rightarrow 0} \int_{(\alpha, \beta) \in \partial \Omega_{\varepsilon}} \mathbf{n} \cdot \nabla y d S=-\lim _{\varepsilon \rightarrow 0} \int_{(\alpha, \beta) \in \Omega_{\varepsilon}} \frac{H_{2}(x, y)}{\varepsilon} d V,
$$

where $d S$ and $d V$ denote the usual surface and volume forms on these respective regions. To compute the limits, we consider a small region around the final curve $L$ which has width $\varepsilon$. We then parameterize this region using new coordinates, $\sigma_{n} \in\left[-\frac{\varepsilon}{2}, \frac{\varepsilon}{2}\right]$ which is locally normal to the curve, and $\sigma_{L} \in L$ which is a dimensional arclength coordinate along the limiting curve. Then the integrals and limits in (24)-(25) are independent of $\sigma_{L}$, and so the limiting behaviour will occur pointwise along $L$. For each such point, we can evaluate the limits as

$$
\begin{gathered}
{\left[d_{1} \mathbf{n} \cdot \nabla x\right]_{-}^{+}=\lim _{\varepsilon \rightarrow 0} \int_{-\frac{\varepsilon}{2}}^{\frac{\varepsilon}{2}} \frac{g(x, y)}{\varepsilon} d \sigma_{n},} \\
{\left[d_{3} \mathbf{n} \cdot \nabla y\right]_{-}^{+}=-\lim _{\varepsilon \rightarrow 0} \int_{-\frac{\varepsilon}{2}}^{\frac{\varepsilon}{2}} \frac{H_{2}(x, y)}{\varepsilon} d \sigma_{n},}
\end{gathered}
$$

where we define the limiting jump condition

$$
[f]_{-}^{+}=\lim _{\varepsilon \rightarrow 0}\left\{f\left(\sigma_{n}=\frac{\varepsilon}{2}\right)-f\left(\sigma_{n}=-\frac{\varepsilon}{2}\right)\right\} .
$$

We identify the jump in the boundary fluxes as the limiting flux along the curve. Finally, applying the Fundamental Theorem of Calculus to the right hand sides of (26)-(27) (as functions of $\sigma_{n}$ ), we obtain the reactive boundary conditions given by (20)(21).

As mentioned above, a detailed spatially continuous model may not be valid for the interactions between bears and salmon, depending on the spatial scales and number of individuals involved, but we find this setting illustrative of the approach. 
One alternative population interaction with similar geometric complexity would be competing bacterial phenotypes within biofilms, for which a continuum model may be more appropriate (Nadell et al., 2016). Reaction-diffusion models have also been successfully applied to insect invasions and other settings wherein dispersal may occur over regions of different spatial scales, and hence simplifying such boundaries may be appropriate there (Hopper and Roush, 1993).

\subsection{Hybrid modeling approaches}

We now discuss more general hybrid modeling approaches. These models can be derived via similar arguments to the reactive boundaries in the previous section, where some region is dimensionally reduced via a limiting process while maintaining the same total rate of population interactions. This approach can result in combinations of features of the continuum and stepping-stone models. Typically, at least one species will be assumed to disperse in a spatially continuous domain and will be modeled by a partial differential equation, and at least one species will be modeled either at discrete nodes or in one spatial dimension only. For the purposes of presenting a general framework, we will assume that $x$ is the species that is modeled in a reduced dimension domain and that $s$ shares the same domain as $y$. As with many features of our models, other specifications are possible depending on the ecological situation of interest.

Let $\Omega$ be a one-dimensional or two-dimensional domain inhabited by $s$ and $y$ and let $\Omega$ be connected to components $\Omega_{1}, \Omega_{2}, \ldots, \Omega_{N}$. The predator and secondary resource are modeled in the continuum setting and the prey population is divided into sub-populations $x^{(1)}, x^{(2)}, \ldots, x^{(N)}$ which reside at the components $\Omega_{1}, \Omega_{2}, \ldots, \Omega_{N}$, respectively. Let $L_{1}, \ldots, L_{N}$ denote the respective parts of $\partial \Omega_{1}, \ldots, \partial \Omega_{N}$ which share boundaries with $\Omega$ and suppose they are parameterized by $\gamma$. The equations are then

$$
\begin{gathered}
\frac{d x^{(j)}}{d t}=f^{(j)}\left(x^{(j)}\right)-g^{(j)}\left(x^{(j)}, Y^{(j)}\right)+\sum_{i=1}^{N}\left(A_{(i, j)} x^{(i)}-A_{(j, i)} x^{(j)}\right) \\
\frac{\partial s}{\partial t}=d_{2} \Delta s+F(s, \alpha, \beta)-G(s, y) \\
\frac{\partial y}{\partial t}=d_{3} \Delta y+H_{1}(s, y)-\delta y
\end{gathered}
$$

where $j=1, \ldots, N$ and $Y^{(j)}$ is defined as

$$
Y^{(j)}=\int_{L_{j}} y d \gamma .
$$

The boundary conditions are given by

$$
\begin{array}{rc}
\mathbf{n} \cdot \nabla s=0, & (\alpha, \beta) \in \partial \Omega, \\
-d_{3} \mathbf{n} \cdot \nabla y=H_{2}\left(x^{(j)}, y\right), & (\alpha, \beta) \in L_{j}, \quad j=1, \ldots, N, \\
\mathbf{n} \cdot \nabla y=0, & (\alpha, \beta) \in \partial \Omega \backslash \bigcup_{j=1}^{N} L_{j},
\end{array}
$$

which represent no-flux conditions on species $s$ and species $y$ away from regions where species $x$ lives, and reactive conditions on these boundaries representing the consumption of species $x$ by $y$.

Another version of the hybrid model represents the situation where the prey is able to move in one spatial dimension along the boundary of $\Omega$, where $\Omega$ is two-dimensional. Let $L$ be a segment of the curve $\partial \Omega$ along which the prey resides. Suppose that $\alpha=\alpha_{0}$ along $L$ and $L$ is parameterized by $\gamma$. Then, the resulting hybrid partial differential equation for $x$ is

$$
\frac{\partial x}{\partial t}=d_{1} \frac{\partial^{2} x}{\partial \gamma^{2}}+f(x, \gamma)-g(x, y) \text {. }
$$

Let $\beta_{1}, \beta_{2}$ be the two endpoints of $L$. Then the no flux and reactive boundary conditions become

$$
\begin{gathered}
\frac{\partial x}{\partial \beta}=0, \quad \beta=\beta_{1}, \beta_{2} \\
\mathbf{n} \cdot \nabla s=0, \quad(\alpha, \beta) \in \partial \Omega, \\
-d_{3} \mathbf{n} \cdot \nabla y=H_{2}(x, y), \quad(\alpha, \beta) \in L, \\
\mathbf{n} \cdot \nabla y=0, \quad(\alpha, \beta) \in \partial \Omega \backslash L .
\end{gathered}
$$

Reactive boundary conditions akin to what we have obtained here have been explored in other settings, such as Maciel and Kraenkel (2014), who relate the population flux and density by mixed boundary conditions in order to model habitat loss. However, in that case a PDE model was directly reduced to an ODE model through a process akin to coarse graining, whereas the reactive boundary conditions here connect populations present in different regions within our model wherein the populations can vary spatially.

In what follows, we will apply these distinct modeling approaches to two specific case studies of ecological relevance.

\section{Case Study 1: Beavers, wolves and white-tailed deer in Voyageurs National Park}

Voyageurs National Park in northern Minnesota is home to a large population of wolves (Canis lupus). White-tailed deer (Odocoileus virginianus) make up a large part of wolf diets and beavers (Castor canadensis) act as a secondary prey, particularly in the ice-free season (Gogan et al., 2004; Johnston, 2017; Gable et al., 2018). Beavers characteristically build dams in streams and rivers which result in so-called beaver ponds (Windels, 2017). Colonies of beavers live in lodges inside these beaver ponds which provide access to forests for food and protection from predators.

Voyageurs National Park has a large range of habitats and population interactions, and we do not hope to model realistic spatiotemporal dynamics across this range. Rather, we focus on trying to understand plausible behaviours involving predation near a river. What does spatial localization of the beaver population within a river, which may be inaccessible to wolves, do to the long-term stability of species coexistence? Does such spatial localization impact the possibility of limit cycles emerging 
from the dynamics? It turns out that the answers to these questions will vary from one modelling framework to another, independent of quantitative readjustments of parameters. Which modelling framework is appropriate will depend on the density of the populations, but it may also be possible to infer an appropriate framework from the dynamics which are precluded by some approaches.

We consider a rectangular domain flanked by a river containing two beaver dams. We describe the following four models: an ODE model, a stepping-stone model, a continuous dispersal model, and a hybrid continuum-stepping-stone model. We denote the deer (primary prey) by $x$, the beavers (secondary prey) by $s$, and the wolves (predator) by $y$. We do not claim to recapitulate the population dynamics throughout any particular region of Voyageurs National Park; instead, we will use this simplified predator-two-prey example to elucidate differences in model behaviors characteristic of each of these modeling paradigms.

\subsection{ODE model}

Motivated by the ODE model of Yamauchi and Yamamura (2005), we formulate the model

$$
\begin{gathered}
\frac{d x}{d t}=r x\left(1-\frac{x}{k}\right)-\theta\left(\frac{x}{x+s+h}\right) y, \\
\frac{d s}{d t}=q s\left(1-\frac{s}{K}\right)-\psi\left(\frac{s}{x+s+h}\right) y, \\
\frac{d y}{d t}=\left(\frac{\epsilon \theta x+\eta \psi s}{x+s+h}\right) y-\delta y,
\end{gathered}
$$

with initial conditions $x(0)=x_{0}, s(0)=s_{0}, y(0)=y_{0}$. Related models have been studied in various contexts by others (Abrams, 2006; Mougi, 2010). Although we shall not do so here, note that this model may be generalized to include depletion of a common resource for both prey species, which results in a logistic term involving both $x$ and $s$ (Genkai-Kato and Yamamura, 1999; Toyokawa, 2017).

Here $r$ and $q$ are the intrinsic growth rates of the primary prey and secondary prey, respectively, and $k$ and $K$ are the corresponding carrying capacities. The parameter $\theta$ is the maximum rate at which wolves can consume deer and $\psi$ is the maximum rate at which wolves can consume beavers. Parameters $\epsilon$ and $\eta$ are conversion factors of the deer and beavers, respectively, which indicate how much the wolves benefit from each food source. Finally, $h$ is a half saturation parameter and $\delta$ is the wolf mortality rate. We assume that all constants are positive, and that $x_{0}, s_{0}$, and $y_{0}>0$. To simplify notation, let $c=\epsilon \theta-\delta$ and $d=\eta \psi-\delta$. In Table 1 we summarize the values of steady states and parameter regimes where these steady states are feasible and stable to small perturbations (for details, see Appendix A).

For our numerical simulations of the ODE and steppingstone models we used MATLAB's 'ode45' solver. We used absolute and relative tolerances of $10^{-9}$ and we ran the simulations for $10^{4}$ time units. A solution was classified as displaying equilibrium dynamics if, for each species, the mean absolute value of the difference between the population size at the
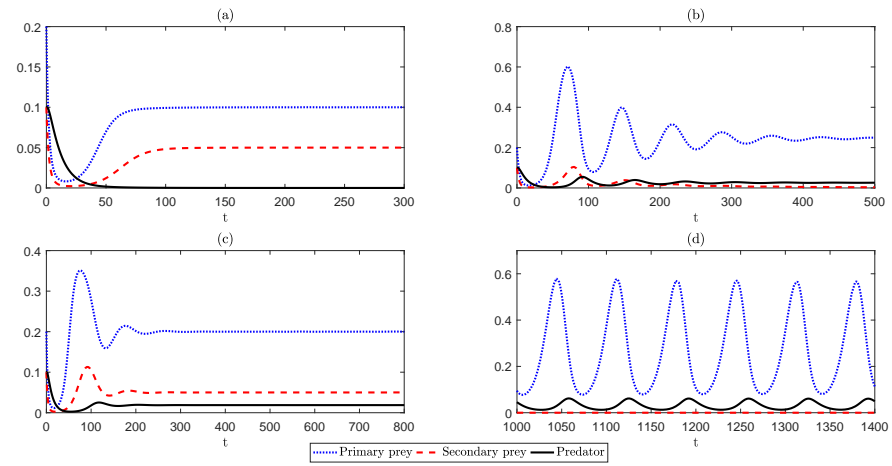

Figure 2: Dynamics of the ODE model (41)-(43) with parameters $r=0.1$, $\theta=5, h=1, q=0.1, \psi=5, \epsilon=0.1, \eta=0.1, \delta=0.1$. The four cases are (a) Predator-free equilibrium: $k=0.1, K=0.05$, (b) Secondary prey-free equilibrium: $k=0.8, K=1$, (c) Positive equilibrium: $k=0.4, K=0.2$, (d) A stable limit cycle: $k=1.6, K=0.6$. Initial conditions are $x(0)=0.2$, $s(0)=0.1, y(0)=0.1$.

last time step and the population size averaged over the last $10^{3}$ time steps was less than $10^{-4}$. The solution was classified as a non-equilibrium point otherwise, but the type of nonequilibrium solution was not classified. In all of our time series plots, we observed convergence to a stable limit cycle in the non-equilibrium regime, but other non-equilibrium behavior may be possible. We note that random initial conditions were used $(\mathcal{N}(0,1)$ represents a normally-distributed random variable with mean zero and unit variance), but that our bifurcation diagram was identical across 10 realizations, suggesting that these dynamics are robust.

In Figure 2, we show time series plots for four different pairs of the primary prey carrying capacity $k$ and the secondary prey carrying capacity $K$. Note that we do not observe any primary prey-free region for these parameters. This can be explained by the mutually exclusive necessary conditions

$$
\eta \psi>\delta \quad \text { and } \quad(\psi r-\theta q)(\eta \psi-\delta) K+\theta q \delta h<0
$$

and

$$
\epsilon \theta>\delta \quad \text { and } \quad(\epsilon \theta-\delta)(\psi r-\theta q) k-\theta q \delta h>0,
$$

for the feasibility and stability of the primary prey-free and secondary prey-free equilibria respectively. For the numerical simulations shown in Figure 2, the second of these conditions is satisfied, so we observe secondary prey-free equilibria.

In Figure 3, we see a predator-free equilibrium for $k$ and $K$ sufficiently small. This is consistent with the stability condition $\epsilon \theta k+\eta \psi K<\delta \pi$. As $k$ increases, the positive equilibrium changes to a secondary prey-free equilibrium, owing to the fact that the predator can sustain higher numbers when primary prey numbers are high. We also observe the paradox of enrichment, whereby increasing the carrying capacity of the primary or secondary prey beyond certain threshold values destabilizes the equilibrium. In this parameter regime we satisfy the conditions of Equation (45), so the primary prey is more resilient to predation. Therefore when non-equilibrium behavior results from increasing the secondary prey carrying capacity, increasing the primary prey carrying capacity stabilizes the dynamics, whereas the opposite is not the case. 


\begin{tabular}{|c|c|c|c|c|c|}
\hline Equilibrium & $x^{*}$ & $s^{*}$ & $y^{*}$ & Feasible & Stable \\
\hline $\begin{array}{l}\text { Secondary } \\
\text { prey-free }\end{array}$ & $\delta h / c$ & 0 & $r \epsilon h(c k-\delta h) / k c^{2}$ & $\epsilon \theta>\delta$ and $k>\delta h / c$ & $\begin{array}{c}\quad(\psi r-\theta q) c k>\psi r \delta h \\
\text { and } r\left(1-2 x^{*} / k\right) \pi^{2}<\theta h y^{*}\end{array}$ \\
\hline $\begin{array}{l}\text { Primary } \\
\text { prey-free }\end{array}$ & 0 & $\delta h / d$ & $q \eta h(d k-\delta h) / k d^{2}$ & $\eta \psi>\delta$ and $K>\delta h / d$ & $\begin{array}{c}(\theta q-\psi r) d K>\theta q \delta h \\
\text { and } q\left(1-2 s^{*} / K\right) \pi^{2}<\psi h y^{*}\end{array}$ \\
\hline Predator-free & $k$ & $K$ & 0 & Always & $\epsilon \theta k+\eta \psi K<\delta \pi$ \\
\hline Positive & $x^{*}$ & $s^{*}$ & $y^{*}$ & $\begin{array}{c}(\eta \psi-\delta) /(\epsilon \theta-\delta)>0 \text { and } \\
0<\frac{\frac{\delta h}{c}-k\left(1-\frac{\theta q}{\psi r}\right)}{\frac{d}{c}+\frac{k \theta q}{\psi r K}}<\min \left\{K, \frac{\delta h}{d}\right\}, \\
(\eta \psi-\delta) /(\epsilon \theta-\delta)<0 \text { and } \\
\frac{\delta h-k\left(1-\frac{\theta q}{\psi}\right)}{\frac{\frac{d}{c}+\frac{k \theta q}{\psi r}}{\psi r K}}>\max \left\{0, \frac{\delta h}{d}\right\}\end{array}$ & $\begin{array}{l}\omega_{1}, \omega_{3}>0 \\
\omega_{1} \omega_{2}>\omega_{3}\end{array}$ \\
\hline
\end{tabular}

Table 1: Feasibility and stability criteria of steady states for the system (41)-(43).

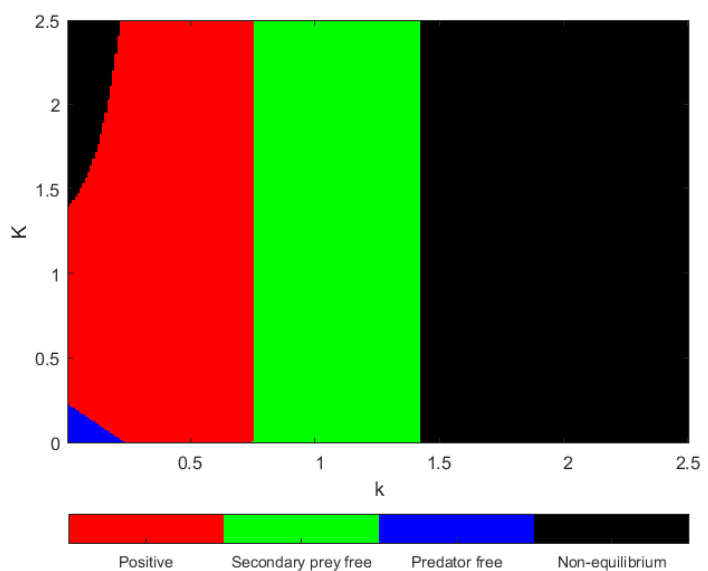

Figure 3: Bifurcation diagram showing the impact of varying the primary prey carrying capacity $(k)$ and the secondary prey carrying capacity $(K)$ on steady state solutions to the ODE model (41)-(43). Parameter values are $r=0.15$, $\theta=5, h=1, q=0.1, \psi=5, \epsilon=0.1, \eta=0.1, \delta=0.1$ and initial conditions are $x(0)=0.02+0.02 \mathcal{N}(0,1), s(0)=0.01+0.01 \mathcal{N}(0,1), y(0)=0.01+0.01 \mathcal{N}(0,1)$. Non-equilibrium dynamics correspond to limit cycles or other unsteady behaviors.

\subsection{Stepping-stone model}

In the stepping-stone model, we use three nodes to represent the regions accessible to the wolves, and two to represent beaver dams. We label the patches with dams as patches 1 and 2, and the patch on land as patch 3 . We consider two cases, depending on whether or not we consider movement of the beavers. For a first version, we assume that the only species able to travel between nodes are the wolves. Beavers are confined to the two dams and deer populate node 3 only. However, we assume wolves are only able to travel across land and not rivers, so there is no wolf migration between nodes 1 and 2. Therefore we have two edges connecting the dams to node 3 to represent the movement of the wolf population. See Figure $4 \mathrm{a}$ for a schematic diagram.

A system of six ordinary differential equations models the dynamics between the populations at the three nodes. We assume logistic growth of the beaver and deer populations in the absence of predation. We have

$$
\frac{d x}{d t}=r x\left(1-\frac{x}{k}\right)-\theta\left(\frac{x}{x+h}\right) y^{(3)}
$$

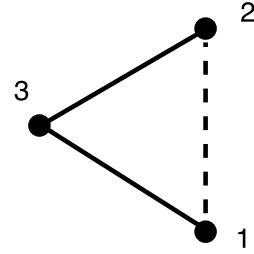

(a)

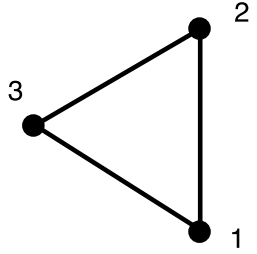

(b)
Figure 4: (a) Stepping-stone network of beavers, wolves and deer on three inter-connected nodes with wolf movement only. Dashed lines denote the edges of the domain and solid lines denote edges which wolves can move along. (b) Stepping-stone network of beavers, wolves and deer on three inter-connected nodes with wolf and beaver movement. Beavers may travel between nodes 1 and 2 and the other edges are for wolf travel only.

$$
\frac{d s^{(j)}}{d t}=q^{(j)} s^{(j)}\left(1-\frac{s^{(j)}}{K^{(j)}}\right)-\psi\left(\frac{s^{(j)}}{s^{(j)}+H}\right) y^{(j)}, j=1,2,
$$

$\frac{d y^{(j)}}{d t}=\left\{\begin{array}{l}\left(\frac{\eta \psi s^{(j)}}{s^{(j)}+H}\right) y^{(j)}-\delta^{(j)} y^{(j)}+\lambda^{(3, j)} y^{(3)}-\lambda^{(j, 3)} y^{(j)}, j=1,2 \\ \left(\frac{\epsilon \theta x}{x+h}\right) y^{(j)}-\delta^{(j)} y^{(j)}+\sum_{i=1}^{3}\left(\lambda^{(i, j)} y^{(i)}-\lambda^{(j, i)} y^{(j)}\right), j=3 .\end{array}\right.$

The initial conditions are $x(0)=x_{0}, s^{(1)}(0)=s_{0}^{(1)}, s^{(2)}(0)=s_{0}^{(2)}$, $y^{(1)}(0)=y_{0}^{(1)}, y^{(2)}(0)=y_{0}^{(2)}, y^{(3)}(0)=y_{0}^{(3)}$. The summation accounts for migration of the predator between nodes $i$ and $j$. Note that $\lambda^{(i, i)}=0$ for $i=1,2,3$, as there is no migration within a patch.

We can also extend the stepping-stone model to allow the movement of beavers between dams via the river. This introduces a further edge to connect nodes 1 and 2, along which only the beavers can move. See the schematic diagram presented in Figure $4 b$.

These additional edges leave the $x$ and $y$ equations unchanged, and change the $s$ equations in the following way:

$\frac{d s^{(1)}}{d t}=q^{(1)} s^{(1)}\left(1-\frac{s^{(1)}}{K^{(1)}}\right)-\psi\left(\frac{s^{(1)}}{s^{(1)}+H}\right) y^{(1)}+\Lambda^{(2,1)} s^{(2)}-\Lambda^{(1,2)} s^{(1)}$, 


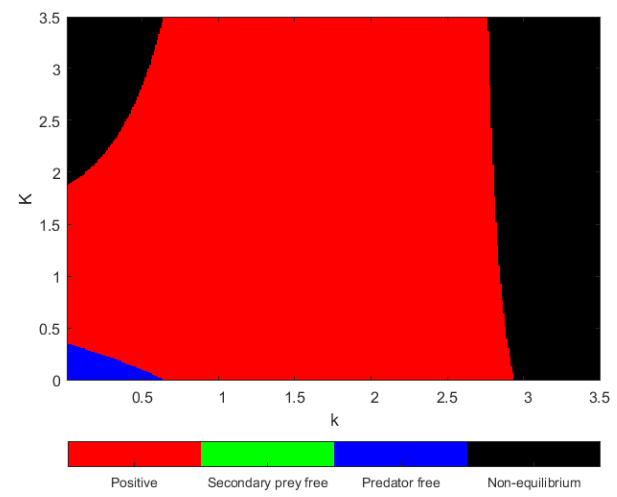

(a) $\lambda=0.1$

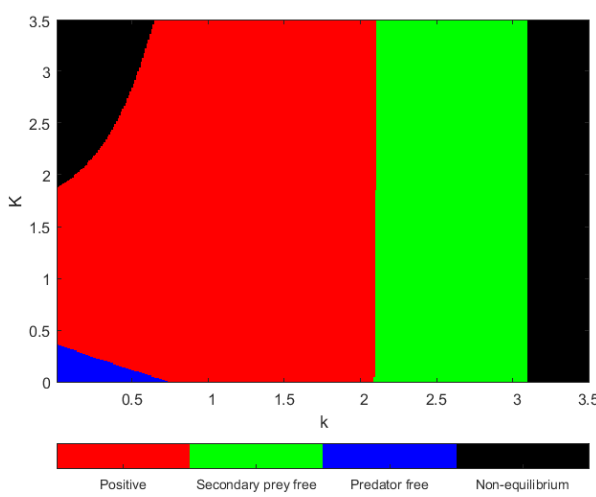

(b) $\lambda=0.2$

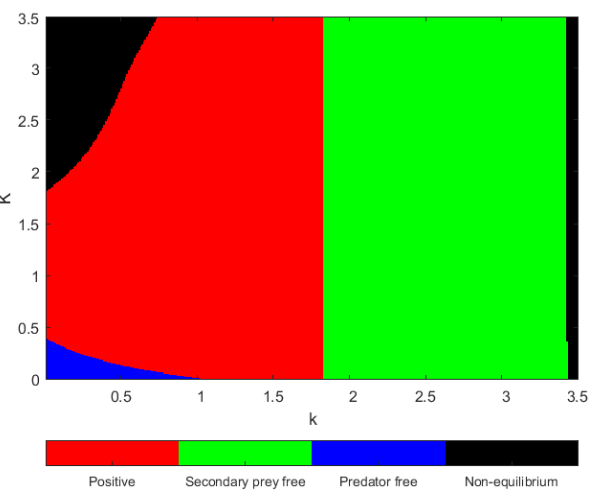

(c) $\lambda=0.5$

Figure 5: Bifurcation diagram showing the impact of varying the primary prey carrying capacity $(k)$ and the secondary prey carrying capacity $(K)$ in the steppingstone model (46)-(48) corresponding to Figure 4a with predator migration rate (a) $\lambda=0.1$, (b) $\lambda=0.2$, (c) $\lambda=0.5$. Other parameter values are $r=0.15, \theta=5$, $h=1, q^{(1)}=q^{(2)}=0.1, K^{(1)}=K^{(2)}=K, \psi=5, H=1, \epsilon=0.1, \eta=0.1, \delta^{(1)}=\delta^{(2)}=\delta^{(3)}=0.1$ and initial conditions are $x(0)=0.02+0.02 \mathcal{N}(0,1)$, $s^{(1)}(0)=s^{(2)}(0)=0.01+0.01 \mathcal{N}(0,1), y^{(1)}(0)=y^{(2)}(0)=y^{(3)}(0)=0.01+0.01 \mathcal{N}(0,1)$.

$\frac{d s^{(2)}}{d t}=q^{(2)} s^{(2)}\left(1-\frac{s^{(2)}}{K^{(2)}}\right)-\psi\left(\frac{s^{(2)}}{s^{(2)}+H}\right) y^{(2)}+\Lambda^{(1,2)} s^{(1)}-\Lambda^{(2,1)} s^{(2)}$

Note that we have used symmetric adjacency matrices $(\lambda$ and $\Lambda)$ corresponding to an undirected graph to represent two way passage between nodes. An alternative model might use a nonsymmetric adjacency matrix for the beavers, corresponding to beavers swimming in only one direction, for example.

In our numerical simulations of the stepping-stone models, the classification of a solution as an equilibrium or nonequilibrium solution is the same as in the ODE case, where now the size of the predator population at time $t$ is averaged over the three nodes:

$$
y(t)=\frac{y^{(1)}(t)+y^{(2)}(t)+y^{(3)}(t)}{3},
$$

and the size of the secondary prey population at time $t$ is averaged over the two nodes representing dams:

$$
s(t)=\frac{s^{(1)}(t)+s^{(2)}(t)}{2} .
$$

We carry out simulations of the stepping-stone model corresponding to the diagram in Figure $4 \mathrm{a}$ and produce bifurcation diagrams examining the impact of varying the primary prey and secondary prey carrying capacities $k$ and $K$ for a variety of predator migration rates, $\lambda^{(i, j)}$, shown in Figure 5. We assume that $\lambda^{(i, j)}$ is constant across the network, so we set $\lambda^{(i, j)}=\lambda$.

Comparing the stepping-stone model bifurcation diagram, Figure 5a, with the ODE model bifurcation diagram, Figure 3, we notice that introducing inter-patch migration at a low rate results in the loss of the secondary prey-free equilibrium, and a wider region of positive equilibrium dynamics, corresponding to coexistence of all three species. This effect arises because migration forces the predator to spend less time at the dams, lessening the impact of predation on the secondary prey. However, when the inter-patch migration rate is increased further, the secondary prey-free region reappears. For large $k$, migration is a good strategy for the predator. Since the predator has to travel via the primary prey to get from one dam to another, by migrating, it will spend more time feeding on primary prey, which can sustain a higher population than the secondary prey. This in turn leads to a predator increase which the secondary prey cannot withstand.

As $\lambda$ increases, the predator-free region stretches out along the $k$ axis for small values of $K$ and, to a lesser extent, along the $K$ axis for small values of $k$. This is because there is nothing to gain from moving to empty patches. The effect is stronger along the $k$ axis, because if $K=0$ then two out of three patches are empty and the effect is weaker along the $K$ axis, since if $k=0$ there is just one empty patch.

Bifurcation diagrams for the stepping-stone model corresponding to the diagram in Figure $4 \mathrm{~b}$, with both predator and secondary prey migration, are very similar those in Figure 5, so they are omitted. It appears that introducing the migration of the secondary prey does not have a significant effect on the long-term dynamics since the predator is equally likely to be at either of the dams. A network without the symmetry between nodes 1 and 2 would be more affected by the introduction of secondary prey migration, but we do not pursue this here.

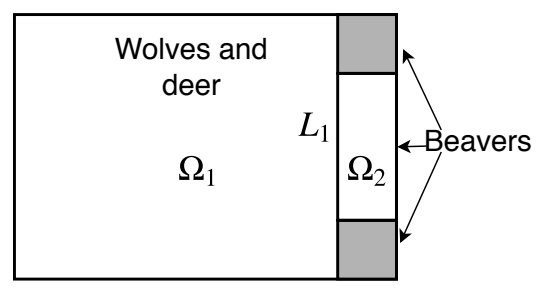

Figure 6: Beaver, wolf, and deer habitat in Voyageurs National Park.

\subsection{Continuum model}

We consider a rectangular domain $\Omega=\left[0, L_{0}\right] \times\left[0, L_{1}\right]$ composed of several disjoint regions depicted in Figure 6 . The spatially continuous model consists of three partial differential 

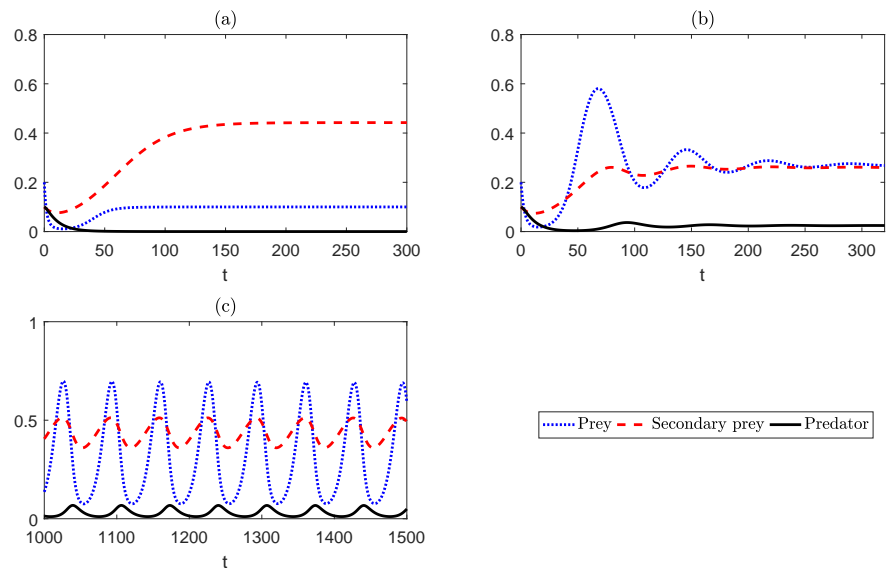

Figure 7: Dynamics of the continuum model (53)-(64) with parameters $d_{1}=$ $d_{2}=d_{3}=0.1, r=0.15, \theta=5, h=1 q=0.1, \psi=5, H=1, \epsilon=0.1, \eta=0.1$, $\delta=0.1$. Initial conditions are $x(0)=0.2, s(0)=0.1, y(0)=0.1$. We observe (a) A predator-free equilibrium: $k=0.1, K=1$, (b) A positive equilibrium: $k=0.75, K=1$, (c) Coexistence in a stable limit cycle: $k=1.7, K=1.8$.

equations

$$
\begin{array}{ll}
\frac{\partial x}{\partial t}=d_{1} \Delta x+r x\left(1-\frac{x}{k}\right)-\theta\left(\frac{x}{x+h}\right) y, & (\alpha, \beta) \in \Omega_{1}, \\
\frac{\partial s}{\partial t}=d_{2} \Delta s+q s\left(I(\alpha, \beta)-\frac{s}{K}\right), & (\alpha, \beta) \in \Omega_{2}, \\
\frac{\partial y}{\partial t}=d_{3} \Delta y+\left(\frac{\epsilon \theta x}{x+h}\right) y-\delta y, & (\alpha, \beta) \in \Omega_{1},
\end{array}
$$

where we define

$$
I(\alpha, \beta)= \begin{cases}1, & \text { if }(\alpha, \beta) \in \Omega_{0}, \\ 0, & \text { if }(\alpha, \beta) \notin \Omega_{0} .\end{cases}
$$

Here $\Omega_{0}$ is the combined region of the two dams, indicated by the shaded regions in Figure 6.

The function $I$ models the fact that the beaver population cannot grow outside $\Omega_{0}$. Deer, beavers, and wolves are allowed to disperse with diffusion rates $d_{1}, d_{2}$, and $d_{3}$, but beavers are confined to the rectangle encompassing the dams and river, and the deer and wolves are confined to the larger adjacent rectangle of land. This represents beavers which may leave their dams and enter the river to forage for food, and wolves which are able to come up to the edge of the river to hunt the beaver, but cannot swim in the river itself (Gable et al., 2016). The boundary conditions are

$$
\begin{gathered}
\mathbf{n} \cdot \nabla x=0, \quad(\alpha, \beta) \in \partial \Omega_{1}, \\
\mathbf{n} \cdot \nabla s=0, \quad(\alpha, \beta) \in \partial \Omega_{2} \backslash L_{1}, \\
-d_{2} \mathbf{n} \cdot \nabla s=-\psi\left(\frac{s}{s+H}\right) y, \quad(\alpha, \beta) \in L_{1}, \\
-d_{3} \mathbf{n} \cdot \nabla y=\left(\frac{\eta \psi s}{s+H}\right) y, \quad(\alpha, \beta) \in L_{1}, \\
-\mathbf{n} \cdot \nabla y=0, \quad(\alpha, \beta) \in \partial \Omega_{1} \backslash L_{1} .
\end{gathered}
$$

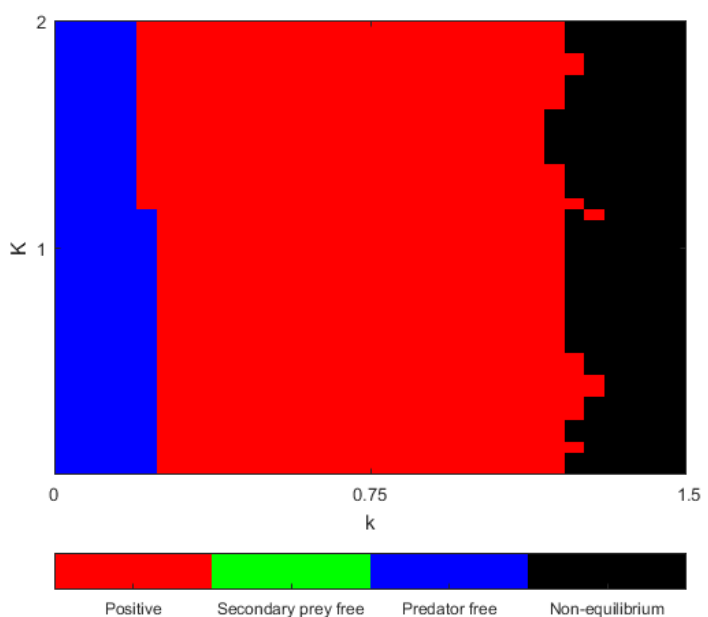

Figure 8: Bifurcation diagram showing the impact of varying the primary prey carrying capacity $(k)$ and the secondary prey carrying capacity $(K)$ in the continuum model (53)-(64) with parameters $r=0.15, \theta=5, h=1, q=0.1, \psi=5$, $H=1, \epsilon=0.1, \eta=0.1, \delta=0.1, d_{1}=d_{2}=d_{3}=0.1$, and initial conditions $x(0)=0.2, s(0)=0.1, y(0)=0.1$.

The initial conditions are

$$
\begin{array}{ll}
x(\alpha, \beta, t=0)=x_{0}(\alpha, \beta), & (\alpha, \beta) \in \Omega_{1}, \\
s(\alpha, \beta, t=0)=s_{0}(\alpha, \beta), & (\alpha, \beta) \in \Omega_{2}, \\
y(\alpha, \beta, t=0)=y_{0}(\alpha, \beta), & (\alpha, \beta) \in \Omega_{1} .
\end{array}
$$

We simulated the continuum model using the coefficient form PDE interface of the commercially-available finiteelement software COMSOL. We defined coefficient form PDEs for the primary prey $x$ and predator $y$ on a $1.5 \times 1$ rectangle, $\Omega_{1}$, and coupled these with a coefficient form PDE for the secondary prey $s$ on an adjacent $0.5 \times 1$ rectangle, $\Omega_{2}$. We used domain probes to measure the mean sizes of the three populations across their respective domains. That is, we obtained time series of the integrals

$$
\begin{aligned}
& \bar{x}(t)=\int_{\Omega_{1}} x(\alpha, \beta, t) d \alpha d \beta, \\
& \bar{s}(t)=\int_{\Omega_{2}} s(\alpha, \beta, t) d \alpha d \beta, \\
& \bar{y}(t)=\int_{\Omega_{1}} y(\alpha, \beta, t) d \alpha d \beta .
\end{aligned}
$$

In Figure 7, we plot the solutions $\bar{x}(t), \bar{s}(t)$, and $\bar{y}(t)$ for three different pairs of the primary prey carrying capacity $k$ and secondary prey carrying capacity $K$. In Figure 8, we carry out a parameter sweep to obtain a bifurcation diagram showing the impact of varying the prey carrying capacity $(k)$ and the secondary prey carrying capacity $(K)$. We used 3,230 triangular mesh elements to discretize the domain, and integrated the equations forward in time using a tolerance of $10^{-4}$. As before, we computed the solution for $10^{4}$ time units. A solution was classified as an equilibrium point if, for each species, the mean absolute value of the difference between the (spatial) mean value of the population at the last time step and this spatial mean averaged 


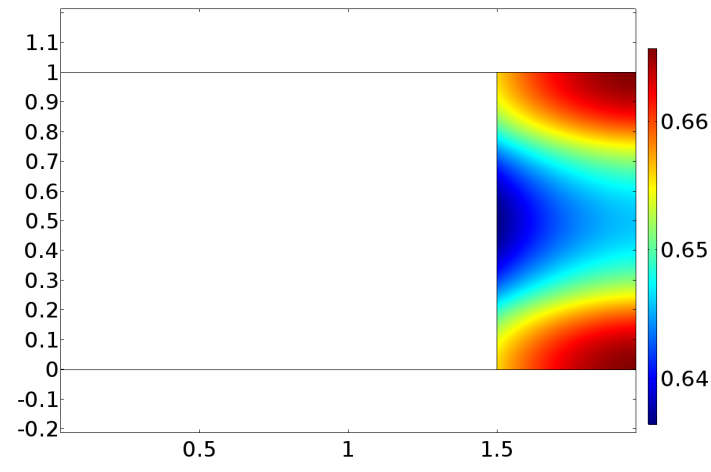

(a) Secondary prey

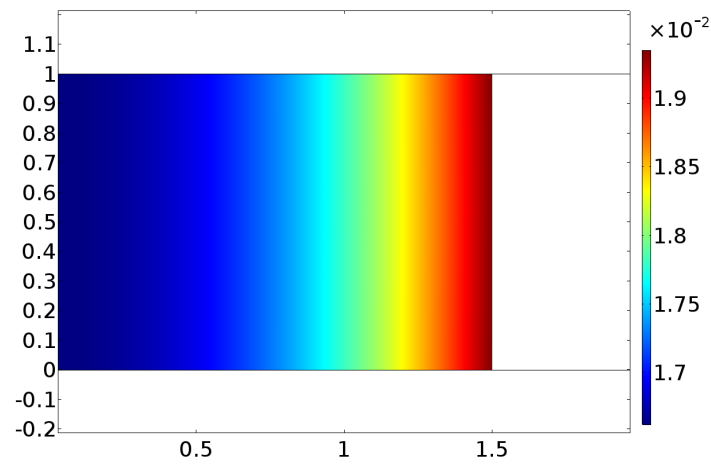

(b) Predator

Figure 9: Spatial spread of predator and secondary prey at positive equilibrium in the continuum model (53)-(64) with parameters as in Figure 8 and $k=0.4$, $K=2$.

over the last $10^{3}$ time steps was less than $10^{-4}$. The solution was classified as a non-equilibrium point otherwise; as in the discrete case, we only observed limit cycle non-equilibrium dynamics but cannot rule out other behaviors such as chaos.

From Figure 8, we observe that a secondary prey-free equilibrium, which was present in the ODE model, does not occur in the continuum model. This is the same effect we saw in the stepping-stone model with low migration rate, where movement allowed the predator to better utilize both food sources, so that less pressure was put on the secondary prey population. Although it is not immediately clear in Figure 8, we did not observe non-equilibrium behavior for large values of the secondary prey carrying capacity and small values of the primary prey carrying capacity. This is due to the fact that the predator and secondary prey are never mixed together; they only interact at a boundary.

Spatial plots of the prey and predator at positive equilibrium in Figure 9 show clustering of the prey away from the boundary of the river where it experiences predation. The prey populations are highest in the corners of each dam, since these are the areas of prey reproduction which are furthest from predation. As we would expect, the predator gravitates towards the nutritionally dense river edge, and its population gradient gradually decreases to its lowest at the opposite side of the region. We note that varying the dispersal rates and other parameters can accentuate or dampen the spatial heterogeneity of each population, but that the spatial population structure typically has the same qualitative spatial variation as shown.

\subsection{Hybrid continuum-reduced-dimensional model}

One can think of the width of the river as being thin, and approximate it as a one dimensional domain along the boundary of the region where the wolves and deer move. Such a hybrid model consists of a partial differential equation for the movement of the wolves and deer in two spatial dimensions, and a partial differential equation for the movement of the beavers between the two dams in one spatial dimension. The river is assumed to be much more narrow than the land, therefore in this model it is flattened to a line. We introduce a reactive boundary condition at the right-hand edge of the domain to reflect the uptake of beavers from the dams. Suppose that the right-hand edge corresponds to $\alpha=\alpha_{2}$. See Figure 10 for a schematic diagram.

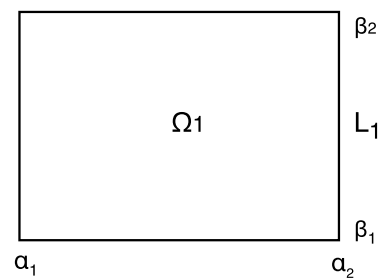

Figure 10: Hybrid model in which the beaver domain is modeled as a line.

We then have the following hybrid system:

$$
\begin{array}{ll}
\frac{\partial x}{\partial t}=d_{1} \Delta x+r x\left(1-\frac{x}{k}\right)-\theta\left(\frac{x}{x+h}\right) y, & (\alpha, \beta) \in \Omega_{1}, \\
\frac{\partial s}{d t}=d_{2} \frac{\partial^{2} s}{\partial \beta^{2}}+q s\left(1-\frac{s}{K}\right)-\psi\left(\frac{s}{s+H}\right) y\left(\alpha_{2}, \beta\right), \beta \in L_{1}, \\
\frac{\partial y}{\partial t}=d_{3} \Delta y+\left(\frac{\epsilon \theta x}{x+h}\right) y-\delta y, & (\alpha, \beta) \in \Omega_{1} .
\end{array}
$$

The boundary conditions are

$$
\begin{gathered}
-\mathbf{n} \cdot \nabla x=0, \quad(\alpha, \beta) \in \partial \Omega_{1}, \\
-\mathbf{n} \cdot \nabla y=\left(\frac{\eta \psi s}{s+H}\right) y, \quad(\alpha, \beta) \in L_{1}, \\
\mathbf{n} \cdot \nabla y=0, \quad(\alpha, \beta) \in \partial \Omega_{1} \backslash L_{1} .
\end{gathered}
$$

The initial conditions are

$$
\begin{aligned}
x(\alpha, \beta, t=0) & =x_{0}(\alpha, \beta), & (\alpha, \beta) \in \Omega_{1}, \\
s(\beta, t=0) & =s_{0}(\beta), & \beta \in\left[\beta_{1}, \beta_{2}\right], \\
y(\alpha, \beta, t=0) & =y_{0}(\alpha, \beta), & (\alpha, \beta) \in \Omega_{1} .
\end{aligned}
$$

In the hybrid model, we used the domain probes defined in Equations (65) and (67) to find the average primary prey and predator densities, but since the secondary prey was defined on a line, we used a boundary probe to calculate the average secondary prey density as

$$
\bar{s}(t)=\int_{\beta_{1}}^{\beta_{2}} s(\beta, t) d \beta .
$$




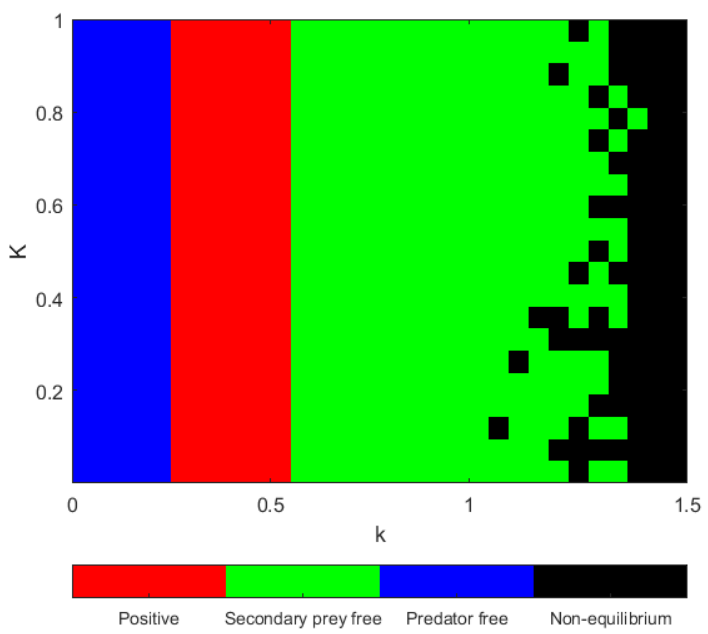

Figure 11: Bifurcation diagram showing the impact of varying the primary prey carrying capacity $(k)$ and the secondary prey carrying capacity $(K)$ in the hybrid model (68)-(76) with parameters as in Figure 8.

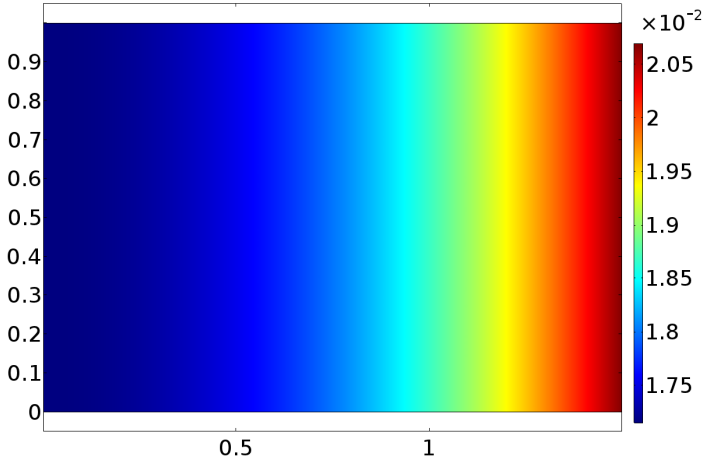

Figure 12: Spatial spread of predator at positive equilibrium in the hybrid model (68)-(76) with parameters as in Figure 8 and $k=0.4, K=2$.

In Figure 11, our bifurcation plot shows that the hybrid model exhibits a secondary prey-free equilibrium region, where the continuum model did not. This is to be expected as the hybrid model prevents the secondary prey from dispersing away from the predator and hence the secondary prey is being eaten in all parts of its domain, rather than at a single boundary. Qualitatively, the appearance of a region of secondary prey-free equilibrium is the main difference between the hybrid and continuum model and we suspect that for higher diffusion rates in the continuum model, the secondary prey-free region would appear. We note that to make a quantitative comparison of the two models, parameters should undergo a length scaling, since $\Omega_{2}$ has changed from a two-dimensional to a one-dimensional domain. This has not been implemented in our simulations as we are primarily interested in qualitative comparisons. We also note that the bifurcation boundaries near the non-equilibrium regimes are rough, and sensitive to the choice of thresholds; in a realistic ecological situation, small differences between behaviors would not be observable due to stochastic fluctuations.

The spatial spread of the predator at the positive equilibrium in the hybrid model, shown in Figure 12, closely resembles the distribution seen in the continuum model, shown in Figure $9 \mathrm{~b}$. This is in spite of the fact that spatially heterogeneous sec-

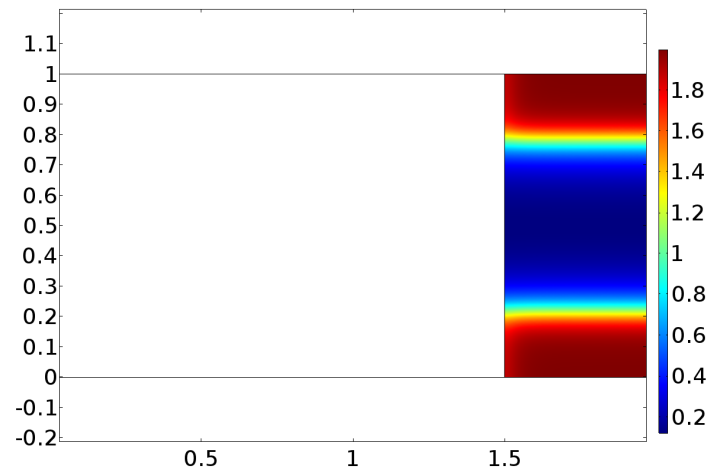

(a) Secondary prey

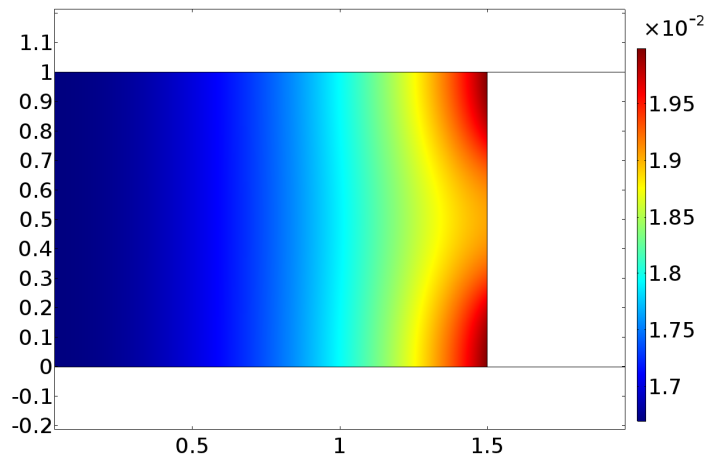

(b) Predator

Figure 13: Spatial spread of predator and secondary prey at positive equilibrium in the continuum model (53)-(64) with parameters as in Figure 8 except for $d_{2}=10^{-4}$ and $k=0.4, K=2$

ondary prey growth was implemented in the continuum model and not in the hybrid model. If the dispersal rate of the secondary prey is substantially reduced, this will not be the case, and the predator distribution in the continuum model will be heterogeneous in $\beta$. We show an example of this in Figure 13, where the predator population varies in $\beta$ only slightly, even for $d_{2}=10^{-4}$. This is due to the predation only occurring along the boundary $L_{1}$, which minimizes the impact that the secondary prey can have on the predator's spatial distribution.

\subsection{Hybrid continuum-stepping-stone model}

Another approach to simplifying the full geometry shown in Figure 6 would be to assume that the dams can be modeled as points, and that the wolves are unable to eat migrating beavers within the river, but can only consume them at these dams. One can then adopt the stepping-stone formalism for the beavers as given by (49)-(50), where the value of the predator at these dams can be given as the local densities $y^{(1)}(t)=y(t, 1.5,0)$ and $y^{(2)}(t)=y(t, 1.5,1)$, and then couple these values at the corners of the geometry given in Figure 10. However, there is a subtle problem in modifying the continuum dynamics, as the approach we have shown in Section 3.1 can only suggest limiting forms of fluxes across boundaries. Nevertheless, applying this argument twice to first reduce the river to a line, and then to reduce the length of a one-dimensional dam to a point, we obtain an expression for the pointwise contribution of the population dynamics at these dams via a Dirac $\delta$-function at the 
relevant corners. We use equation (68) for the primary prey, and write the predator dynamics as

$$
\begin{aligned}
\frac{\partial y}{\partial t}= & d_{3} \Delta y+\left(\frac{\epsilon \theta x}{x+h}\right) y-\delta y+\operatorname{Dirac}_{(1.5,0)}(\alpha, \beta)\left(\frac{\eta \psi s^{(1)}}{s^{(1)}+H}\right) y \\
& +\operatorname{Dirac}_{(1.5,1)}(\alpha, \beta)\left(\frac{\eta \psi s^{(2)}}{s^{(2)}+H}\right) y
\end{aligned}
$$

for $(\alpha, \beta) \in \Omega_{1}$, where we define $\operatorname{Dirac}_{\left(\alpha_{0}, \beta_{0}\right)}(\alpha, \beta)$ to represent the Dirac $\delta$-function centered at the point $\left(\alpha_{0}, \beta_{0}\right)$ in the problem domain. (We avoid the more common $\delta_{\left(\alpha_{0}, \beta_{0}\right)}(\alpha, \beta)$ notation, in order to avoid confusion with the death rate parameters.) Finally, we specify no-flux conditions on all boundaries of the two-dimensional region $\Omega_{1}$.

We remark that this formulation is equivalent to the approach used in Section 3.1, if we had instead placed Dirac $\delta$-functions along the boundary curve $L$, but the derivation there clearly explains the limiting flux. Here it is more complicated to think of 'flux' reactions coming from a point in two spatial dimensions. Nevertheless, simulations of this model give qualitatively similar behaviour to the full continuum model, with some spatial localization as one might expect; see Figure 14, and compare it with Figure 13b. Note that the quantitative differences here are due both to different choices of parameters, and to the models being somewhat incomparable (as there is no predation along the rightmost boundary, but it is instead localized at these corners). The spatial structure of solutions is qualitatively similar across the parameter ranges we have considered, with the main qualitative difference arising due to this effect of spatial localization. As with the continuum and the other hybrid model, this approach does not lead to non-equilibrium dynamics for large beaver carrying capacities, suggesting that the spatial localization in all three models involving only boundary or pointwise interactions between species, does not permit sustained oscillations. As in the previous hybrid model, beaver-free equilibria exist if the value of $k$ is sufficiently large.

\section{Case Study 2: Bears, berries and salmon on Kodiak Is- land}

High densities of Kodiak Brown Bear (Ursus arctos middendorffi) inhabit Kodiak Island in the Gulf of Alaska. Their primary food source is Pacific Salmon (Oncorhynchus spp.) (Barnes Jr, 1990; Reimchen, 2000), while they consume berries as an allochthonous resource (Berns and Hensel, 1972; Berns et al., 1980; Willson, 1993). As with the previous case study, we are interested in the possible dynamics emergent from different choices of how to model nontrivial spatial structure. As an exemplar region, we consider a rectangular domain with a river running along the right-hand side. To the left of the river is an area of land where berries grow. While such a caricature region may be overly simplistic to understand the population dynamics of the bears across the whole island, it provides a useful setting in which to observe qualitative differences between modelling approaches.

We will model the populations of bear (predator), salmon (prey), and berry (allochthonous resource or subsidy) in the

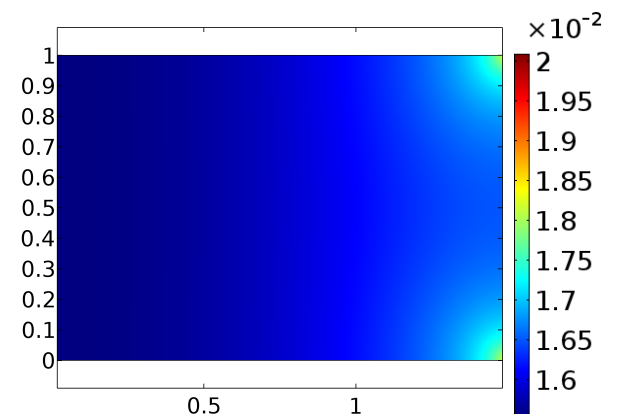

(a)

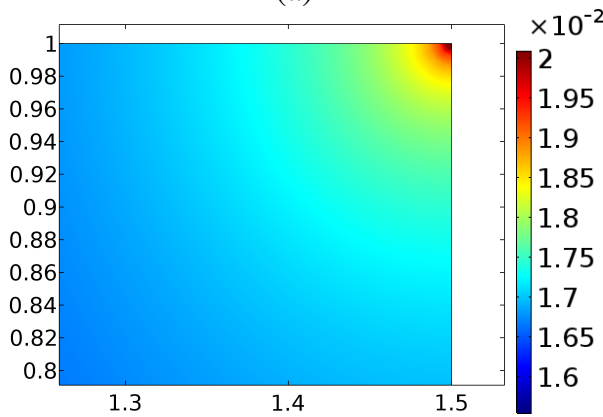

(b)

Figure 14: Spatial spread of predator at positive equilibrium in the hybrid model given by (49)-(50), (68), and (78) with parameters as in Figure 13b. In (a) we view the whole domain whereas in (b) we show the distribution near the second prey point at the corner.

following settings: an ODE model at one point in space, a stepping-stone model, a continuous dispersal model, and two distinct hybrid continuum-stepping-stone models. Let $x, s$, and $y$ denote the population densities of the salmon, berries, and bears, respectively.

\subsection{ODE model}

The simplest model ignores spatial effects entirely and consists of a system of three ODEs at a single point. We have the system

$$
\begin{gathered}
\frac{d x}{d t}=r x\left(1-\frac{x}{k}\right)-\theta\left(\frac{x}{x+s+h}\right) y, \\
\frac{d s}{d t}=i-b s-\psi\left(\frac{s}{x+s+h}\right) y, \\
\frac{d y}{d t}=\left(\frac{\epsilon \theta x+\eta \psi s}{x+s+h}\right) y-\delta y,
\end{gathered}
$$

with initial conditions $x(0)=x_{0}, s(0)=s_{0}, y(0)=y_{0}$. Here $r$ is the intrinsic growth rate of the prey and $k$ is the carrying capacity. The parameter $\theta$ is the maximum rate at which bears can consume salmon and $\psi$ is the maximum rate at which bears can consume berries. Parameters $\epsilon$ and $\eta$ are conversion factors of the salmon and berries respectively, which indicate how much the bears benefit from each food source. Finally, $h$ is a half saturation parameter, $i$ is the constant berry input rate, $\mathrm{b}$ is the rate of berry decay, and $\delta$ is the bear mortality rate. It is assumed that all constants are positive, and that $x_{0}, s_{0}$, and $y_{0}>0$. 


\begin{tabular}{cccccc}
\hline Equilibrium & $x^{*}$ & $s^{*}$ & $y^{*}$ & Feasible & Stable \\
\hline Subsidy-only & 0 & $i / b$ & 0 & Always & Never \\
\hline Predator-free & $k$ & $i / b$ & 0 & Always & $k / \underline{k}+i / b \underline{l}<1$ \\
\hline Prey-free & 0 & $\underline{l}$ & $(\eta / \delta)(i-b \underline{l})$ & $i>b \underline{l}$ & $i>i^{*}$ \\
\hline Positive & $x^{*}$ & \multirow{2}{*}{$s^{*}$} & $y^{*}$ & $\begin{array}{c}k>\underline{k} \text { and } 0<i<i^{*} \\
k<\underline{k} \text { and } i_{*}(k)<i<i^{*}\end{array}$ & $\begin{array}{c}\omega_{1}, \omega_{3}>0, \\
\omega_{1} \omega_{2}>\omega_{3}\end{array}$ \\
\hline
\end{tabular}

Table 2: Feasiability and stability of steady states solution to the model (79)-(81).

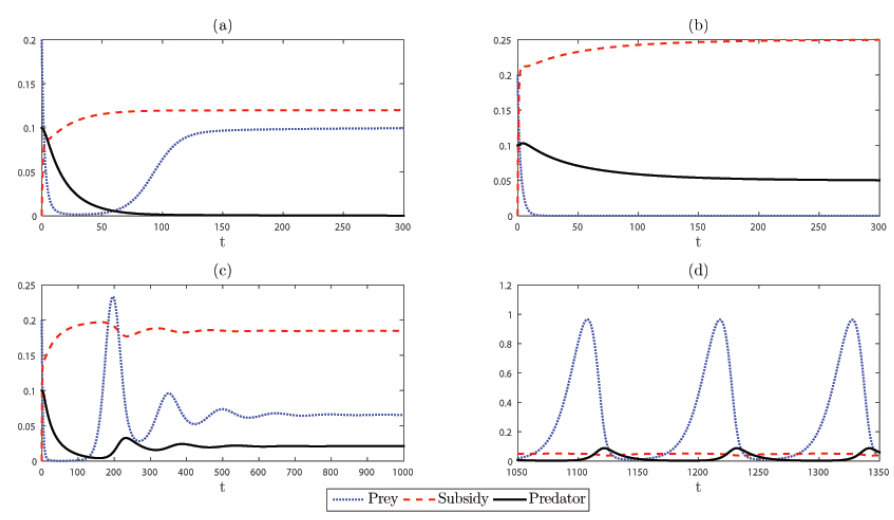

Figure 15: Dynamics of the ODE model (79)-(81) with parameters $r=0.1$, $\theta=5, h=1, b=1, \psi=5, \epsilon=0.1, \eta=0.1, \delta=0.1$. We observe four cases: (a) predator-free equilibrium: $k=0.1, i=0.12$, (b) prey-free equilibrium: $k=0.1$, $i=0.3$, (c) positive equilibrium: $k=0.4, \mathrm{i}=0.2$ and (d) Stable limit cycle: $k=2, i=0.05$. Initial conditions are $x(0)=0.2, s(0)=0, y(0)=0.1$.

For the predator-prey-subsidy ODE model in Section 5, we are able to find steady states and investigate local stability analytically as in Nevai and Van Gorder (2012). Since our ODE model is a relabeling of their model, we do not reproduce their analysis here. A summary of the steady state feasibility and stability is provided in Table 2. In this table, we make use of the quantities $\underline{k}=\frac{\delta h}{\epsilon \theta-\delta}, \underline{l}=\frac{\delta h}{\eta \psi-\delta}, i^{*}=\frac{\delta h(b+(r \psi / \theta))}{\eta \psi-\delta h}, i_{*}(k)=$ $\left(1-\frac{k}{k}\right) \frac{\delta h}{\eta \psi-\delta}, \omega_{1}=-\operatorname{tr}(J), \omega_{2}=J_{11} J_{22}-\left(J_{12} J_{21}+J_{13} J_{31}+J_{23} J_{32}\right)$, $\omega_{3}=-\operatorname{det}(J)$, where $J$ is the Jacobian of the system (79)-(81).

We again use MATLAB to produce time series plots of the solution to the ODE model for four different pairs of prey carrying capacity $k$ and allochthonous resource or subsidy input rate $i$, shown in Figure 15. We use the same numerical procedures and parameters as in Section 4 unless stated otherwise.

The $(k, i)$ bifurcation diagram supports our analytical predictions for the predator-free and prey-free equilibrium regions. As in Table 2, the predator-free region is both feasible and stable for $k<(1-i / b l) \underline{k}$, which is precisely the lower left region that appears in Figure 16. As expected biologically, a higher allochthonous resource availability reduces the predator's reliance on the prey. This is reflected in the boundary of the stability region, $k=(1-i / b \underline{l}) \underline{k}$, which is a decreasing function of $i$. The prey-free region is both feasible and stable for $i>(b+r \psi / \theta) \underline{l}$, which is precisely the prey-free region we see in Figure 16. It is intuitive that the boundary of the prey-free region is an increasing function of the prey growth rate $r$, as the predator must be better subsidized to drive a faster growing prey population to extinction.

In the lower right corner of Figure 16 we see the shape of the

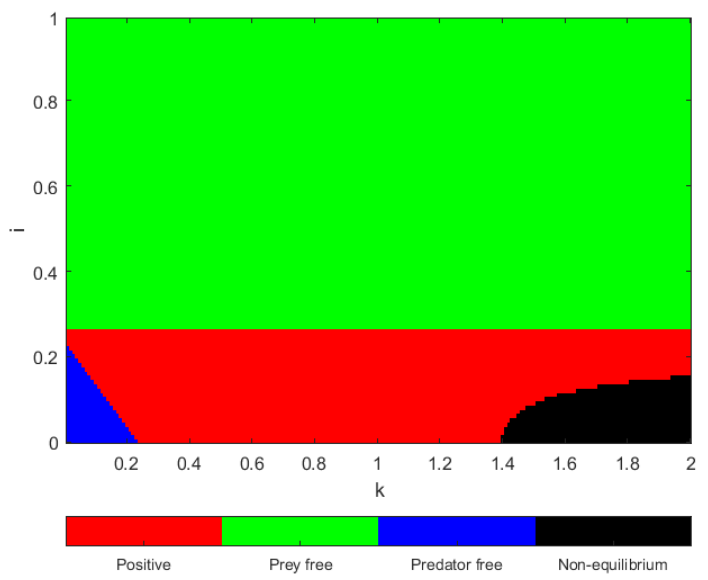

Figure 16: Bifurcation diagram showing the impact of varying the prey carrying capacity $(k)$ and the allochthonous resource input rate $(i)$ in the ODE model (79)-(81). Parameter values are $r=0.1, \theta=5, h=1, b=1, \psi=5, \epsilon=0.1$, $\eta=0.1, \delta=0.1$, and initial conditions are $x(0)=0.01+0.01 \mathcal{N}(0,1), s(0)=$ $0.01+0.01 \mathcal{N}(0,1), y(0)=0.01+0.01 \mathcal{N}(0,1)$.

non-equilibrium region, which Nevai and Van Gorder (2012) were unable to determine analytically. The transition from a positive equilibrium to non-equilibrium dynamics as the prey carrying capacity increases is an example of the paradox of enrichment (Roy and Chattopadhyay, 2007). Increasing the allochthonous resource input rate stabilizes the dynamics to a positive equilibrium followed by a prey-free equilibrium, as seen in Nevai and Van Gorder (2012).

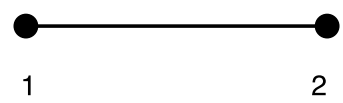

Figure 17: Stepping-stone domain for bears, berries, and salmon on Kodiak Island.

\subsection{Stepping-stone model}

In the stepping-stone model, we can view the domain as a network of two nodes, where berries exist at node 1 only, salmon exist at node 2 only, and bears are able to travel between the two nodes. See the schematic diagram presented in Figure 17.

If we refer to the node on land as node 1 and the node in the river as node 2 and we denote $y^{(j)}$ as the density of bears at node $j$, then the following ODEs describe the population dynamics:

$$
\begin{gathered}
\frac{d x}{d t}=r x\left(1-\frac{x}{k}\right)-\theta\left(\frac{x}{x+h}\right) y^{(2)}, \\
\frac{d s}{d t}=i-b s-\psi\left(\frac{s}{s+H}\right) y^{(1)},
\end{gathered}
$$




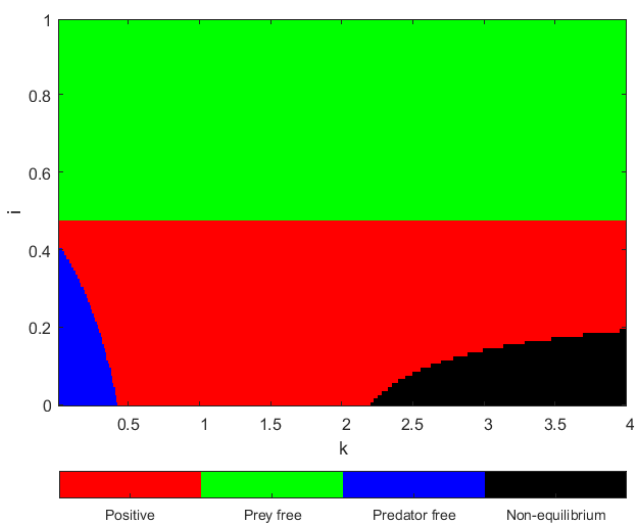

(a) $\lambda=0.1$

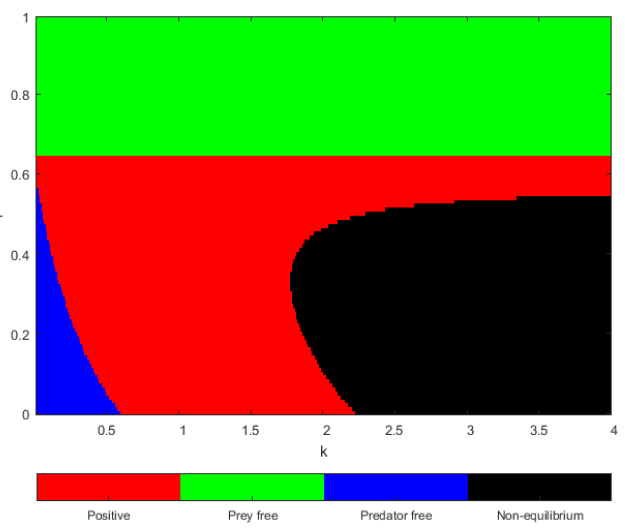

(b) $\lambda=1$

Figure 18: Bifurcation diagram showing the impact of varying the prey carrying capacity $(k)$ and the allochthonous resource input rate $(i)$ in the stepping-stone model (82)-(85) with predator migration rate (a) $\lambda=0.1$, (b) $\lambda=1$. Parameter values are $r=0.1, \theta=5, h=1, b=1, \psi=5, \epsilon=0.1, \eta=0.1, \delta^{(1)}=\delta^{(2)}=0.1$, $\lambda^{(i, j)}=\lambda$, for all $i, j$, and initial conditions are $x(0)=0.01+0.01 \mathcal{N}(0,1), s(0)=0.01+0.01 \mathcal{N}(0,1), y^{(1)}(0)=y^{(2)}(0)=0.01+0.01 \mathcal{N}(0,1)$.

$$
\begin{aligned}
& \frac{d y^{(1)}}{d t}=\left(\frac{\eta \psi s}{s+H}\right) y^{(1)}-\delta^{(1)} y^{(1)}+\lambda^{(2,1)} y^{(2)}-\lambda^{(1,2)} y^{(1)}, \\
& \frac{d y^{(2)}}{d t}=\left(\frac{\epsilon \theta x}{x+h}\right) y^{(2)}-\delta^{(2)} y^{(2)}+\lambda^{(1,2)} y^{(1)}-\lambda^{(2,1)} y^{(2)} .
\end{aligned}
$$

The initial conditions are $x(0)=x_{0}, s(0)=s_{0}, y^{(1)}(0)=y_{0}^{(1)}$, $y^{(2)}(0)=y_{0}^{(2)}$.

In our numerical simulations of the stepping-stone model corresponding to the diagram in Figure 17, our classification of a solution as equilibrium or non-equilibrium was the same as in the ODE case, where now the size of the predator population at time $t$ is averaged over the two nodes:

$$
y(t)=\frac{y^{(1)}(t)+y^{(2)}(t)}{2} .
$$

Motivated by our findings in the non-spatial case, we investigate numerically the $(k, i)$ dependence of the stepping-stone model for different values of the predator inter-patch migration rate $\lambda^{(i, j)}$, which we assume to be constant across the network, $\lambda^{(i, j)}=\lambda$. Bifurcation plots are shown in Figure 18. Increasing $\lambda$ does not have a qualitative effect on the dynamics, but we do observe quantitative differences between the three bifurcation diagrams. First note that introducing inter-patch migration increases the threshold value of $k$ at which non-equilibrium dynamics invade the positive equilibrium.

In the ODE model, limit cycles first appear at approximately $k=1.4$ for small values of $i$, but in the stepping-stone models, non-equilibrium behavior is not seen until approximately $k=2.2$ (for $\lambda=0.1$ ). As predator movement increases, the minimum prey carrying capacity which can support a positive non-subsidized predator population increases and the minimum allochthonous resource input rate that can support the predator when prey is scarce increases. This is due to the energy wasted by moving unnecessarily to the empty patch. We also observe that an increased inter-patch migration rate leads to a smaller prey-free region. This mirrors the results obtained in Nevai and Van Gorder (2012) and suggests that increasing the predator migration rate decreases the time the predator is able to spend consuming its preferred resource. In contrast to Nevai and Van Gorder (2012), where resources are limited in one of the patches, we observe that increasing the predator migration rate increases the size of the non-equilibrium region. Destabilization due to migration in the presence of abundant food sources has been observed in Shen and Van Gorder (2017) for a larger network structure (a complete graph on ten nodes).

\subsection{Continuum model}

The continuous dispersal model allows bears and berries to disperse throughout the central rectangle and salmon to disperse in the river. See Figure 19 for a schematic diagram.

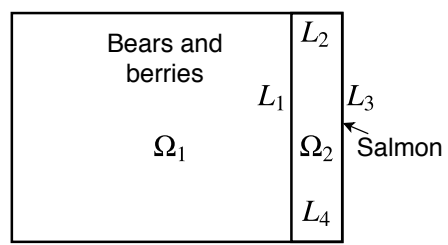

Figure 19: Bears, berries, and salmon in a rectangular domain with a river along the right-hand side.

The system is modeled by the three PDEs

$$
\begin{gathered}
\frac{\partial x}{\partial t}=d_{1} \Delta x+r x\left(1-\frac{x}{k}\right),(\alpha, \beta) \in \Omega_{0}, \\
\frac{\partial s}{\partial t}=d_{2} \Delta s+i-b s-\psi\left(\frac{s}{s+H}\right) y,(\alpha, \beta) \in \Omega_{1}, \\
\frac{\partial y}{\partial t}=d_{3} \Delta y+\left(\frac{\eta \psi s}{s+H}\right) y-\delta y,(\alpha, \beta) \in \Omega_{1},
\end{gathered}
$$

with boundary conditions

$$
\mathbf{n} \cdot \nabla x=0, \quad(\alpha, \beta) \in L_{3},
$$



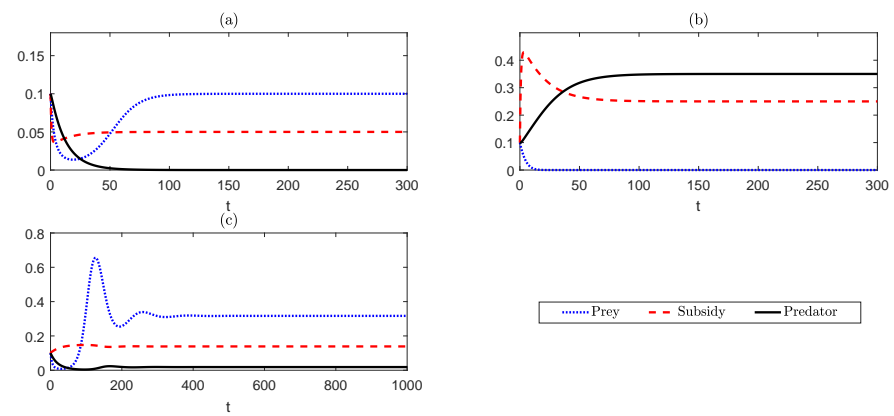

Figure 20: Dynamics of the continuum model (87)-(99) with parameters $r=$ $0.1, \theta=20, h=1, b=1, \psi=5, H=1, \epsilon=0.1, \eta=0.1, \delta=0.1, \kappa=0$, $d_{1}=d_{3}=0.1$, and $d_{2}=0.001$. Initial conditions are $x(0)=0.1, s(0)=0.1$, $y(0)=0.1$. We observe three cases: (a) A predator-free equilibrium: $k=0.1$, $i=0.12$, (b) A prey-free equilibrium: $k=1, i=0.6$, (c) A positive equilibrium: $k=1, i=0.15$.

$$
\begin{array}{cc}
-\mathbf{n} \cdot \nabla x=-\theta\left(\frac{x}{x+h}\right) y, & (\alpha, \beta) \in L_{1}, \\
-\mathbf{n} \cdot \nabla x=\kappa_{i}, & (\alpha, \beta) \in L_{2}, \\
-\mathbf{n} \cdot \nabla x=-\kappa_{o} x, & (\alpha, \beta) \in L_{4}, \\
\mathbf{n} \cdot \nabla s=0, & (\alpha, \beta) \in \partial \Omega_{1}, \\
-\mathbf{n} \cdot \nabla y=\left(\frac{\epsilon \theta x}{x+h}\right) y, & (\alpha, \beta) \in L_{1}, \\
-\mathbf{n} \cdot \nabla y=0, & (\alpha, \beta) \in \partial \Omega_{1} \backslash L_{1},
\end{array}
$$

and initial conditions

$$
\begin{array}{ll}
x(\alpha, \beta, t=0)=x_{0}(\alpha, \beta), & (\alpha, \beta) \in \Omega_{0}, \\
s(\alpha, \beta, t=0)=s_{0}(\alpha, \beta), & (\alpha, \beta) \in \Omega_{1}, \\
y(\alpha, \beta, t=0)=y_{0}(\alpha, \beta), & (\alpha, \beta) \in \Omega_{1} .
\end{array}
$$

The allochthonous resource (berries) is allowed to disperse but naturally at a lower rate than the bears and salmon, so we impose $d_{2} \ll d_{1}, d_{3}$. The constant flux $\kappa_{i}$ at $L_{2}$ represents the inflow of salmon to the river and the flux $-\kappa_{o} x$ at $L_{4}$ accounts for salmon flowing out of the river (in general the rates here need not be equal, but for simplicity we assume $\kappa \equiv \kappa_{i}=\kappa_{o}$ ).

Using COMSOL, as in Section 4, we simulated the continuum model across a range of parameters. We defined PDEs for the allochthonous resource $s$ and predator $y$ on a $1.5 \times 1$ rectangle, $\Omega_{1}$, and coupled these with a PDE for the prey $x$ on an adjacent $0.5 \times 1$ rectangle, $\Omega_{0}$. We used a domain probe to measure the spatial means of the three populations across their respective domains. That is, we calculated the time series

$$
\begin{aligned}
& \bar{x}(t)=\int_{\Omega_{0}} x(\alpha, \beta, t) d \alpha d \beta, \\
& \bar{s}(t)=\int_{\Omega_{1}} s(\alpha, \beta, t) d \alpha d \beta, \\
& \bar{y}(t)=\int_{\Omega_{1}} y(\alpha, \beta, t) d \alpha d \beta .
\end{aligned}
$$

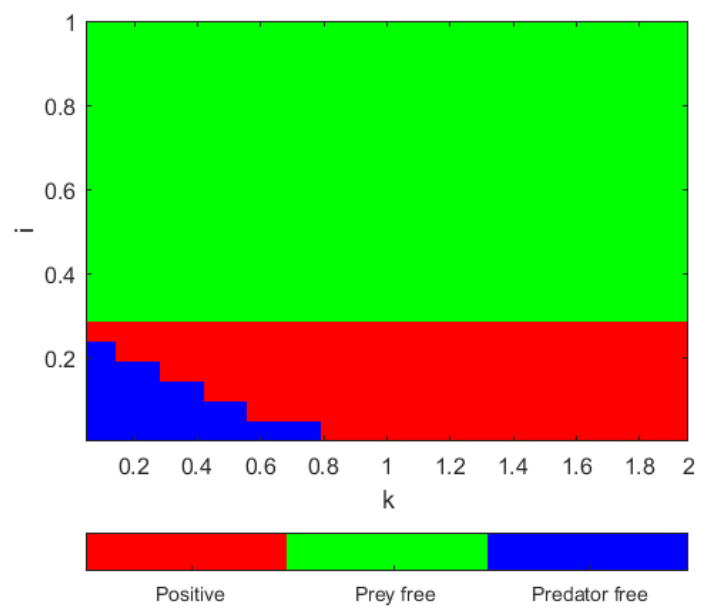

Figure 21: Bifurcation diagram showing the impact of varying the prey carrying capacity $(k)$ and the allochthonous resource input rate $(i)$ in the continuum model (87)-(99). Parameter values are $r=0.1, \theta=20, h=1, b=1, \psi=5$, $\epsilon=0.1, \eta=0.1, \delta=0.1, \kappa=0, d_{1}=d_{3}=0.1, d_{2}=0.001$, and initial conditions are $x(0)=0.1, s(0)=0.1, y(0)=0.1$.

In Figure 20, we plot the solutions $\bar{x}(t), \bar{s}(t)$, and $\bar{y}(t)$ for three different pairs of the primary prey carrying capacity $k$ and allochthonous resource input rate $i$. In Figure 21, we carry out a parameter sweep to obtain a bifurcation diagram showing the impact of varying the prey carrying capacity $(k)$ and the allochthonous resource input rate $(i)$. A solution was classified as an equilibrium point if, for each species, the mean absolute value of the difference between the probe value at the last time step and the probe data averaged over a set of the last time steps was less than $10^{-4}$. The solution was classified as a nonequilibrium point otherwise.

In contrast with the discrete models, we do not observe any non-equilibrium dynamics in the continuum model. This is due to the fact that the predator and prey now occupy separate domains, so they can only interact at the boundary where their two domains meet. The predator and allochthonous resource on the other hand do mix in the same domain, however the positive equilibrium in a pure predator-subsidy system is never unstable (Nevai and Van Gorder, 2012). Other than this, the continuum model has the same qualitative features as its discrete counterparts.

The continuum model allows us to observe the spatial distribution of the three species over time. In Figure 22, we observe how, at equilibrium, the predator has higher density at the boundary of the river, gradually decreasing to its lowest value at the left-hand border of the domain. The opposite is true for the prey: $x$ is highest on the boundary furthest from the river and is lowest beside the river where it experiences predation.

\subsection{Hybrid continuum-stepping-stone model}

A hybrid model will combine features of both the steppingstone model and the continuum model. Experimental data indicates that at Karluk Lake on Kodiak Island, the home range for brown bears is at least $0.5 \mathrm{~km}$ and could be up to $49.2 \mathrm{~km}$ (Berns et al., 1980). Since the bears and berries naturally spread over a wider space relative to the salmon, we permit dispersal of bears 


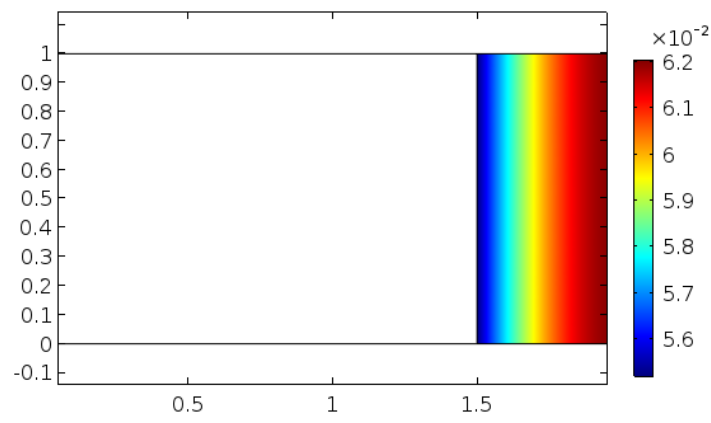

(a) Prey

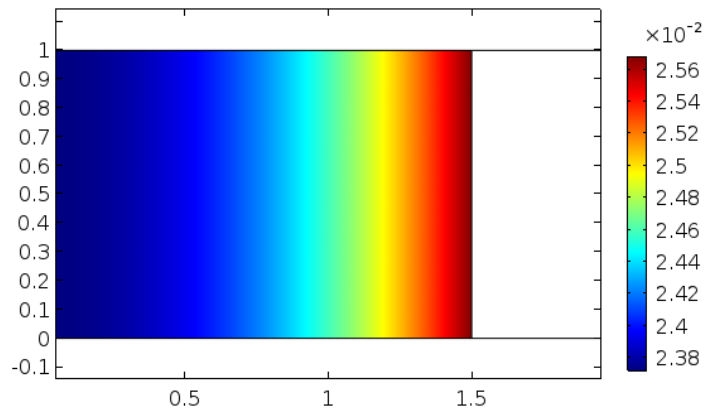

(b) Predator

Figure 22: Spatial spread of predator and allochthonous resource at equilibrium in the continuum model (87)-(99) with parameters as in Figure 21 and $k=0.6$, $i=0.25$.

and berries on land but model the salmon population with a reduced dispersal region. See Figure 23.
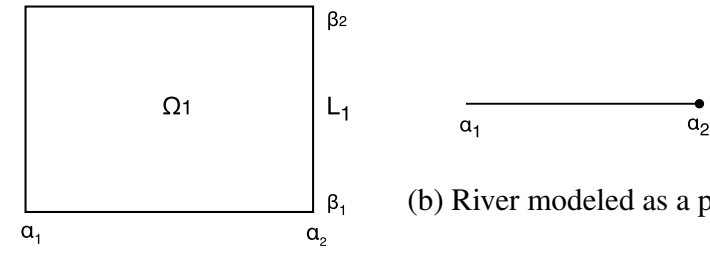

(b) River modeled as a point

(a) River modeled as a line

Figure 23: Two possible domains for hybrid continuum-stepping-stone model.

In Figure 23a, $L_{1}$ is the line which divides the river from the land. In this case, we have the following system of PDEs:

$$
\begin{gathered}
\frac{\partial x}{\partial t}=d_{1} \frac{\partial^{2} x}{\partial \beta^{2}}+r x\left(1-\frac{x}{k}\right)-\theta\left(\frac{x}{x+h}\right) y\left(\alpha_{2}, \beta\right), \beta \in L_{1}, \\
\frac{\partial s}{\partial t}=d_{2} \Delta s+i-b s-\psi\left(\frac{s}{s+H}\right) y,(\alpha, \beta) \in \Omega_{1}, \\
\frac{\partial y}{\partial t}=d_{3} \Delta y+\left(\frac{\eta \psi s}{s+H}\right) y-\delta y,(\alpha, \beta) \in \Omega_{1} .
\end{gathered}
$$

The boundary conditions are

$$
\begin{array}{cc}
\frac{\partial x}{\partial \beta}=-\kappa_{o} x, & \beta=\beta_{1}, \\
\frac{\partial x}{\partial \beta}=\kappa_{i}, & \beta=\beta_{2},
\end{array}
$$

$$
\begin{gathered}
\mathbf{n} \cdot \nabla s=0, \quad(\alpha, \beta) \in \partial \Omega_{1}, \\
-\mathbf{n} \cdot \nabla y=\left(\frac{\epsilon \theta x}{x+h}\right) y, \quad(\alpha, \beta) \in L_{1}, \\
\mathbf{n} \cdot \nabla y=0, \quad(\alpha, \beta) \in \partial \Omega_{1} \backslash L_{1} .
\end{gathered}
$$

The initial conditions are

$$
\begin{aligned}
x(\beta, t=0)=x_{0}(\beta), & \beta \in\left[\beta_{1}, \beta_{2}\right], \\
s(\alpha, \beta, t=0)=s_{0}(\alpha, \beta), & (\alpha, \beta) \in \Omega_{1}, \\
y(\alpha, \beta, t=0)=y_{0}(\alpha, \beta), & (\alpha, \beta) \in \Omega_{1} .
\end{aligned}
$$

The domain shown in Figure 23b is formed by removing the $\beta$ axis from Figure 23a. This simplification is suitable when conditions do not vary in $\beta$, for example when $\kappa=0$ (as before, we set $\kappa \equiv \kappa_{o}=\kappa_{i}$ ). The equations reduce to an ODE for $x$ and partial differential equations for $s$ and $y$ :

$$
\frac{d x}{d t}=r x\left(1-\frac{x}{k}\right)-\theta\left(\frac{x}{x+h}\right) y\left(\alpha_{2}\right),
$$

$$
\begin{gathered}
\frac{\partial s}{\partial t}=d_{2} \frac{\partial^{2} s}{\partial \alpha^{2}}+i-b s-\psi\left(\frac{s}{s+H}\right) y, \alpha \in\left[\alpha_{1}, \alpha_{2}\right], \\
\frac{\partial y}{\partial t}=d_{3} \frac{\partial^{2} y}{\partial \alpha^{2}}+\left(\frac{\epsilon \theta x}{x+h}\right) y+\left(\frac{\eta \psi s}{s+H}\right) y-\delta y, \alpha \in\left[\alpha_{1}, \alpha_{2}\right] .
\end{gathered}
$$

The boundary conditions are

$$
\begin{array}{cc}
\frac{\partial s}{\partial \alpha}=0, \quad \frac{\partial y}{\partial \alpha}=0, & \alpha=\alpha_{1}, \\
\frac{\partial s}{\partial \alpha}=0, \quad \frac{\partial y}{\partial \alpha}=\left(\frac{\epsilon \theta x}{x+h}\right) y, & \alpha=\alpha_{2} .
\end{array}
$$

The initial conditions are

$$
\begin{array}{cc}
x(0)=x_{0}, & \\
s(\alpha, t=0)=s_{0}(\alpha), & \alpha \in\left[\alpha_{1}, \alpha_{2}\right], \\
y(\alpha, t=0)=y_{0}(\alpha), & \alpha \in\left[\alpha_{1}, \alpha_{2}\right] .
\end{array}
$$

For the two-dimensional hybrid model shown in Figure 23a, we use the domain probes defined in Equations (101) and (102) to find the average allochthonous resource and predator densities, but since the prey is now defined on a line, we use a boundary probe to calculate the average prey density:

$$
\bar{x}(t)=\int_{\beta_{1}}^{\beta_{2}} x(\beta, t) d \beta .
$$

The $(k, i)$ bifurcation plot for the two-dimensional hybrid model is shown in Figure 24a.

In the one-dimensional hybrid model corresponding to Figure $23 \mathrm{~b}$, the averaged allochthonous resource and predator populations are given by the line integrals

$$
\bar{s}(t)=\int_{\alpha_{1}}^{\alpha_{2}} s(\alpha, t) d \alpha,
$$




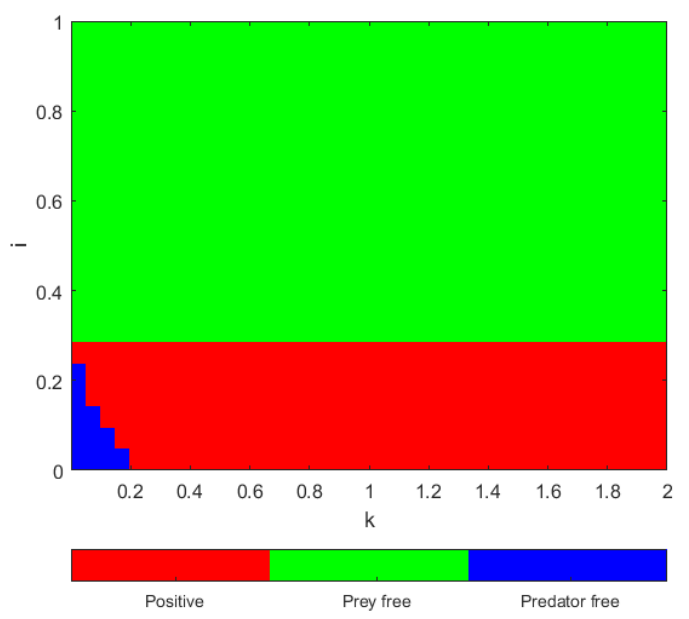

(a) Bifurcation diagram for Figure 23a

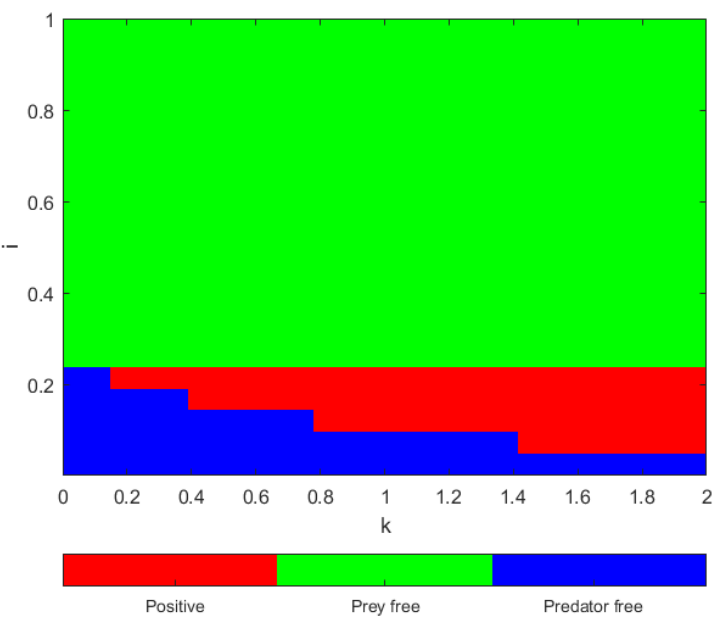

(b) Bifurcation diagram for Figure 23b

Figure 24: Bifurcation diagrams showing the impact of varying the prey carrying capacity $(k)$ and the allochthonous resource input rate $(i)$ in the hybrid continuumstepping-stone model corresponding to the domain in (a) Figure 23a (given by (103)-(113)), (b) 23b (given by (114)-(121)), with $\theta=10$. Parameter values and initial conditions are as in Figure 21.

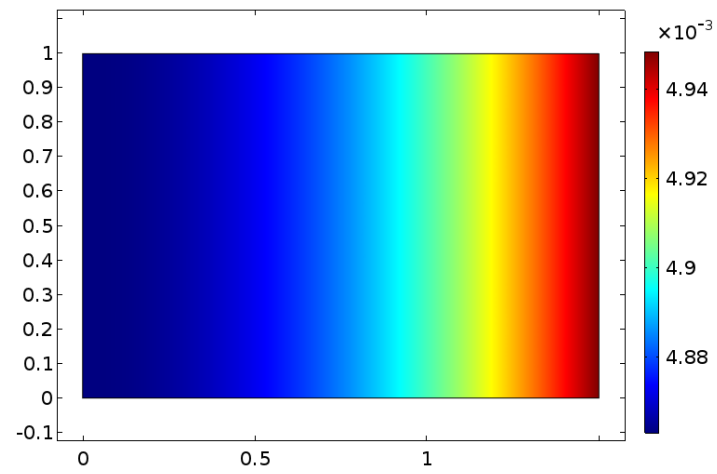

Figure 25: Spatial spread of predator at positive equilibrium in the twodimensional hybrid model with parameters as in Figure 21 and $k=0.6$, $i=0.25$.

$$
\bar{y}(t)=\int_{\alpha_{1}}^{\alpha_{2}} y(\alpha, t) d \alpha .
$$

The $(k, i)$ bifurcation plot for the one-dimensional hybrid model is shown in Figure 24b.

Note that the different scalings of the continuum model and the two hybrid models produce quantitatively different bifurcation boundaries, however all three plots show the same qualitative behavior. This suggests that the reduction of the twodimensional continuum model to a line and an ODE, which is both much simpler mathematically and computationally, is a reasonable approximation. The predator also has the same spatial spread at positive equilibrium in the two-dimensional hybrid model as in the continuum model, shown in Figure 25. Thus we can get an idea of the spatial heterogeneity without needing to solve the full two-dimensional system.

Our analysis thus far has considered the case of no external flow of salmon (e.g. $\kappa=0$ ), corresponding to a fixed body of water, rather than to a flowing river. We now demonstrate spatially heterogeneous solutions in Figure 26 in the case of nonzero inflow and outflow of salmon. For this case, the one dimensional-hybrid model is insufficient to capture the spatial structure of the two-dimensional continuum model, although the two-dimensional hybrid model has qualitatively similar spatial heterogeneity in the bear population to the continuum model.

\section{Discussion}

Considering a hybrid modeling approach to spatial dynamics involving a generalist predator, we have compared ODE, continuum, stepping-stone, and hybrid continuum-stepping-stone models for both of our ecological case studies based on caricature representations of the spatial structure of their habitats. In each of our ODE models, we have derived feasibility and stability criteria for the steady states of the system and outlined how this could also be done for the stepping-stone models. For each model, we have produced bifurcation diagrams showing the long-term population dynamics under a range of parameter regimes and we have used these bifurcation plots to see how the different models compare in their predictions.

In our analysis of the predator-two-prey model, we observe that introducing inter-patch migration at a low rate can have a positive effect on secondary prey populations. When the interpatch migration rate was sufficiently small, secondary prey-free dynamics, seen in the ODE model, were lost and the three species were able to coexist. For high migration rates, this effect disappears and the bifurcation diagram is qualitatively similar to the ODE case. Transitioning from the stepping-stone to the continuum model, we again see the prevalence of the secondary prey for small diffusion of the predators. The approximation of the river by a line in the hybrid model has a negative effect on the secondary-prey population, since they experience predation everywhere in their domain. For this reason, the preyfree steady state is feasible in the hybrid model, even when the predator diffusion is small.

A feature present in all of our predator-two-prey models is a region of non-equilibrium dynamics when the primary-prey 


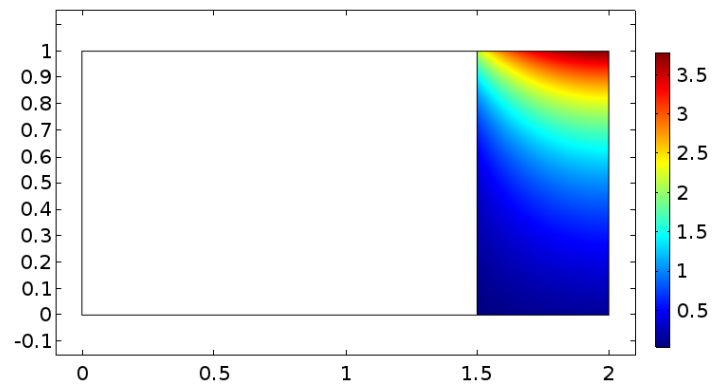

(a) Continuum Prey

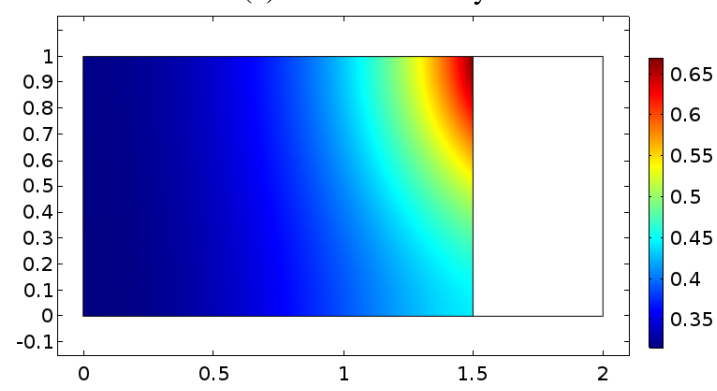

(b) Continuum Predator

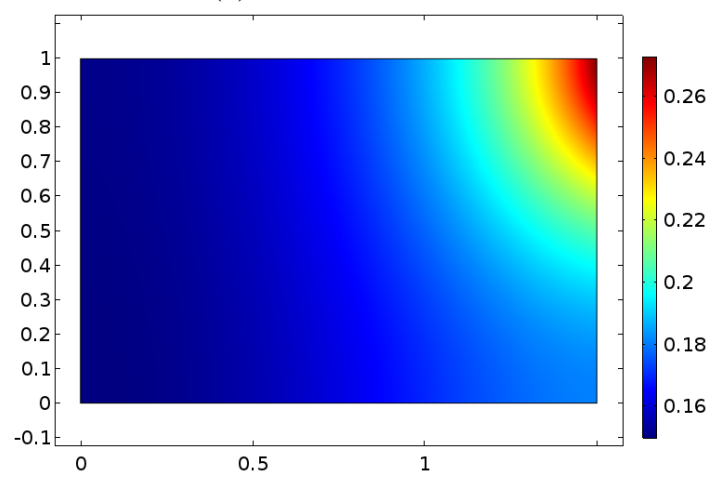

(c) Hybrid Predator

Figure 26: Spatial spread of predator and prey at positive equilibrium in the continuum model in (a) and (b), and the predator in the two-dimensional hybrid model in (c), all with parameters as in Figure 21 and $k=0.6, i=0.25$. We set $\kappa=\kappa_{i}=\kappa_{o}=1$. carrying capacity is sufficiently large, due to the paradox of enrichment. In the ODE and stepping-stone models we also see a region of instability when the secondary prey carrying capacity is large, which can be mitigated by increasing the primary prey carrying capacity. This region is not present in the continuum or hybrid models, because in these models the secondary prey and predator only interact at a boundary. The fact that this spatial localization of the secondary prey precludes the possibility of the predator and prey to become locked in limit cycle dynamics suggests that whether or not these populations can be considered as spatially separated is an important question.

Neglecting the non-equilibrium region for high $K$, the predator-two-prey continuum model with low diffusion is qualitatively similar to the stepping-stone model with low inter-patch migration rate. Provided that the secondary prey (which we assume to be less resilient than the primary prey) has a low carrying capacity and predator movement is slow, we believe that the stepping-stone model is a good approximation to the continuum model. The hybrid model is a poor approximation when diffusion rates are low, because it is precisely the ability of the secondary prey to move around $\Omega_{2}$ which prevents it from becoming extinct. This is demonstrated in the spatial plots of Figure 9 , where the secondary prey is seen to cluster away from the river edge.

When the predator has a high rate of motion, there is little to gain qualitatively from using a stepping-stone model over an ODE model. However, if an ecologist were interested in estimating the value of $k$ at which the prey-free equilibrium appears or is destabilized, the stepping-stone model would be a preferable choice because these bifurcation points vary with the migration rate $\lambda$. For situations with large dispersal rates, the hybrid model produces qualitatively similar results to the stepping-stone models. The key advantage of the hybrid model is that it can be used to visualize the spatial spread of the predator and allochthonous resource. We did not produce a continuum model bifurcation plot for high diffusion rates, but the probe plot for $d_{1}=d_{2}=d_{3}=0.5$ and $k=K=1$ converges to a secondary prey-free equilibrium and we hypothesize that the dynamics would be similar to the hybrid model, again without the non-equilibrium region for large $K$ values. Therefore, the main advantage of using the continuum model, for any diffusion rate, would be to observe the spatial distribution of species in each sub-domain.

In our analysis of the predator-prey-subsidy model, we observe that the stepping-stone model produces a qualitatively similar bifurcation diagram to the ODE model. Quantitatively, the stepping-stone models have smaller prey-free regions than the ODE model, and a higher prey carrying capacity is required to induce instability. We observe three changes in solutions to the stepping-stone model as the predator migration rate increases: for small values of the allochthonous resource input rate, $i$, the predator-free region extends further along the $k$ axis and for small values of the prey carrying capacity, $k$, the predator-free region extends further along the $i$ axis; the secondary prey-free region decreases in size; and we see a larger region of non-equilibrium dynamics. The first of these effects occurs because the predator population decays rapidly while 
dispersing through nutritionally poor patches. The second effect is also a result of the predator's inefficiency; at high migration rates, the predator population cannot aggregate around its preferred food source and hence, without sufficient subsidization, does not grow large enough to kill off the prey. The third effect is a phenomenon similar to the paradox of enrichment, and has been observed to occur when a predator migrates through a network with abundant food sources. However, from the literature, we know that this destabilization effect is highly dependent on the network structure. In a range of other networks, increased movement was found to have an opposite, stabilizing effect (Shen and Van Gorder, 2017).

In our predator-prey-subsidy case study, we see no qualitative differences between the ODE model and the stepping-stone model for any values of the predator migration rate. However, the non-equilibrium region in the discrete models, which grows as the predator migration rate increases, is in stark contrast with the continuum and hybrid models where the domain separation completely eliminates non-equilibrium behavior. This occurs as a result of the strict sub-division of the predator and prey habitats and the fact that non-equilibrium dynamics cannot arise from a simple predator-allochthonous resource interaction (Nevai and Van Gorder, 2012). One may question whether in reality there would exist such a hard boundary between regions $\Omega_{1}$ and $\Omega_{0}$ or whether there could be some level of overlap. A hybrid model where the predator can enter part of $\Omega_{0}$, such as shown in Figure 1, would be an interesting extension to our model. It would be useful to compare such a model with a threenode version of the stepping stone model where the added node is a prey refuge, and the prey travels between the refuge node and the predator-bearing node. Of course, on small scales the continuum assumption needed to use the formalism of partial differential equations may break down as well.

Unlike in the predator-two-prey case study, the predatorprey-subsidy model demonstrates the same qualitative equilibrium behavior for all four model types. Therefore, if one is interested in studying only stable long-time dynamics, each of our models would give a similar qualitative picture, and the ODE model would be the obvious choice for simplicity. However, in order to study instability in the predator-prey-subsidy system, our investigations reveal that a detailed understanding of the crossover between the predator and prey domains is vital. If complete separation is falsely assumed, then non-equilibrium behavior will be missed. On the other hand, an overly patchy model with a fast-moving predator may exaggerate any underlying instability inherent in the model dynamics.

Our analysis may help to distinguish when spatial effects are most likely to have an impact and which types of models can capture the types of spatial influence they are interested in. When choosing a model, one must also take into consideration the field data they have available. It should be noted that, even if a model is theoretically a good fit, if the parameters involved cannot be realistically estimated, the model may have no chance of performing well. The continuum model poses the most challenges in this respect, requiring estimates of diffusion coefficients and multiple length scales. Additionally, such reactiondiffusion models are not always good representations of popu- lations, particularly when the local spatial density is small so that stochastic effects involving individuals come into play.

The motivation of our analysis was the predator-prey-subsidy interaction between bears, salmon, and berries on Kodiak island, and the predator-two-prey interaction between wolves, deer, and beavers in Voyageurs National Park. With suitable parameter and domain adaptations, similar analyses and results could be applied to related interactions in spatially structured environments, such as the interaction between polar bears, ringed seal, and ground-nesting bird colonies in areas of sea ice break-up (Hamilton et al., 2017), or the interaction between golden eagles, feral pigs and island foxes on the California Channel Islands (Roemer et al., 2002). One might also consider the effect of seasonality on the dynamics (Levy et al., 2016; Jansen and Van Gorder, 2018). For example, it has been suggested that beavers are at lower risk of predation in winter months when they spend most of their time inside their lodges buried under a thick layer of snow. However, in early spring, wolves are known to use abandoned beaver lodges as dens for raising pups (Johnston, 2017), so predation of beavers by wolves during this time of year is likely to increase. As in any kind of modeling, there are numerous trade-offs between model complexity and realism that must be made. Balancing simplicity and realism is a crucial part of investigating any ecological phenomena.

\section{Appendix A. Steady state analysis for the system (41)-(43)}

We are not aware of an analysis of the precise model (41)(43) in the literature, so we provide the following feasibility and stability analysis for completeness.

In the predator-two-prey ODE model of Section 4, note that the steady states $\left(x^{*}, s^{*}, y^{*}\right)$ satisfy the following equations:

$$
\begin{gathered}
0=\left[r\left(1-\frac{x^{*}}{k}\right)-\frac{\theta y^{*}}{x^{*}+s^{*}+h}\right] x^{*}, \\
0=\left[q\left(1-\frac{s^{*}}{K}\right)-\frac{\psi y^{*}}{x^{*}+s^{*}+h}\right] s^{*}, \\
0=\left[\frac{\epsilon \theta x^{*}+\eta \psi s^{*}}{x^{*}+s^{*}+h}-\delta\right] y^{*} .
\end{gathered}
$$

We will examine the feasibility and stability of each type of steady state. The feasibility condition amounts to checking that $x^{*}, s^{*}$, and $y^{*}$ are non-negative, since a negative population size is biologically unrealistic. The stability of the steady states will depend on the eigenvalues $\lambda_{1}, \lambda_{2}$, and $\lambda_{3}$ of the Jacobian matrix

$$
J=\left[\begin{array}{lll}
J_{11} & J_{12} & J_{13} \\
J_{21} & J_{22} & J_{23} \\
J_{31} & J_{32} & J_{33}
\end{array}\right],
$$

where

$$
\begin{aligned}
& J_{11}=r\left(1-\frac{2 x^{*}}{k}\right)-\frac{\theta\left(s^{*}+h\right) y^{*}}{\pi^{2}}, \quad J_{12}=\frac{\theta x^{*} y^{*}}{\pi^{2}}, \quad J_{13}=-\frac{\theta x^{*}}{\pi}, \\
& J_{21}=\frac{\psi s^{*} y^{*}}{\pi^{2}}, \quad J_{22}=q\left(1-\frac{2 s^{*}}{K}\right)-\frac{\psi\left(x^{*}+h\right) y^{*}}{\pi^{2}}, \quad J_{23}=-\frac{\psi s^{*}}{\pi},
\end{aligned}
$$




$$
\begin{gathered}
J_{31}=\frac{1}{\pi^{2}}\left(\left(\epsilon \theta\left(s^{*}+h\right)-\eta \psi s^{*}\right) y^{*}\right), \\
J_{32}=\frac{1}{\pi^{2}}\left(\left(\eta \psi\left(x^{*}+h\right)-\epsilon \theta x^{*}\right) y^{*}\right), \quad J_{33}=\frac{\epsilon \theta x^{*}+\eta \psi s^{*}}{\pi}-\delta,
\end{gathered}
$$

and we define $\pi=x^{*}+s^{*}+h$. For linear stability of the steady state, we require $\operatorname{Re}\left(\lambda_{i}\right)<0$, for $i=1,2,3$.

\section{Appendix A.1. Prey-free equilibrium}

Setting $\left(x^{*}, s^{*}, y^{*}\right)=\left(0,0, y^{*}\right)$ implies that $\delta y^{*}=0$, hence the only possible prey-free equilibrium is when all three species are extinct: $\left(x^{*}, s^{*}, y^{*}\right)=(0,0,0)$. The Jacobian matrix has diagonal form

$$
J=\left[\begin{array}{c|cc}
r & 0 & 0 \\
\hline 0 & q & 0 \\
0 & 0 & -\delta
\end{array}\right] .
$$

Since the Jacobian has at least one positive eigenvalue, namely $\lambda_{1}=r$, the prey-free equilibrium is unstable.

\section{Appendix A.2. Secondary prey-free equilibrium}

With $s^{*}=0, x^{*}$ and $y^{*}$ satisfy

$$
r\left(1-\frac{x^{*}}{k}\right)-\frac{\theta y^{*}}{x^{*}+h}=0
$$

or

$$
\frac{\epsilon \theta x^{*}}{x^{*}+h}-\delta=0
$$

Equation (A.6) implies that $x^{*}=\frac{\delta h}{\epsilon \theta-\delta}$, so for feasibility we must have $\epsilon \theta>\delta$. Solving for $y^{*}$ (using (A.5) and this expression for $\left.x^{*}\right)$, we arrive at

$$
y^{*}=\frac{r \epsilon h[(\epsilon \theta-\delta) k-\delta h]}{k(\epsilon \theta-\delta)^{2}} .
$$

Thus for feasibility, we require $(\epsilon \theta-\delta) k-\delta h>0$. Overall we have the secondary prey-free equilibrium

$$
\left(x^{*}, s^{*}, y^{*}\right)=\left(\frac{\delta h}{\epsilon \theta-\delta}, 0, \frac{r \epsilon h[(\epsilon \theta-\delta) k-\delta h]}{k(\epsilon \theta-\delta)^{2}}\right),
$$

which is feasible for $\epsilon \theta>\delta$ and $k>\frac{\delta h}{\epsilon \theta-\delta}$. The corresponding Jacobian matrix is

$$
J=\left[\begin{array}{ccc}
r\left(1-\frac{2 x^{*}}{k}\right)-\frac{\theta h y^{*}}{\pi^{2}} & \frac{\theta x^{*} y^{*}}{\pi^{2}} & -\frac{\theta x^{*}}{\pi} \\
0 & q-\frac{\psi y^{*}}{\pi} & 0 \\
\frac{\epsilon \theta h y^{*}}{\pi^{2}} & \left(\eta \psi\left(x^{*}+h\right)-\epsilon \theta x^{*}\right) \frac{y^{*}}{\pi^{2}} & 0
\end{array}\right],
$$

where $J_{33}=0$ comes from the fact that $\delta\left(x^{*}+h\right)=\epsilon \theta x^{*}$. Clearly, $J_{22}=q-\psi y^{*} / \pi$ is an eigenvalue of $J$, so for stability we require $q \pi-\psi y^{*}<0$. Substituting for $y^{*}$ and rearranging, we arrive at the condition $(\theta q-\psi r)(\epsilon \theta-\delta) k+\psi r \delta h<0$. Denote the other two eigenvalues of $J$ by $\lambda_{1}, \lambda_{2}$. Then $\lambda_{1}, \lambda_{2}$ satisfy $\lambda_{1}+\lambda_{2}=\left(r\left(1-2 x^{*} / k\right) \pi^{2}-\theta h y^{*}\right) / \pi^{2}$ and $\lambda_{1} \lambda_{2}=\epsilon \theta^{2} h x^{*} y^{*} / \pi^{3}>$ 0 . Since $\lambda_{1} \lambda_{2}>0$, for both eigenvalues to be negative, we require $\lambda_{1}+\lambda_{2}<0$ and hence the second stability condition is $r\left(1-2 x^{*} / k\right) \pi^{2}-\theta h y^{*}<0$. Overall, for stability of the secondary prey-free equilibrium, we require

$(\theta q-\psi r)(\epsilon \theta-\delta) k+\psi r \delta h<0 \quad$ and $\quad r\left(1-\frac{2 x^{*}}{k}\right) \pi^{2}-\theta h y^{*}<0$.

\section{Appendix A.3. Primary prey-free equilibrium}

The analysis from Subsection Appendix A.2 follows through for the primary prey-free steady state with a change of notation. The steady state is

$$
\left(x^{*}, s^{*}, y^{*}\right)=\left(0, \frac{\delta h}{\eta \psi-\delta}, \frac{q \eta h[(\eta \psi-\delta) K-\delta h]}{K(\eta \psi-\delta)^{2}}\right),
$$

with corresponding feasibility conditions $\eta \psi>\delta$ and $K>\frac{\delta h}{\eta \psi-\delta}$. The stability conditions are

$(\psi r-\theta q)(\eta \psi-\delta) K+\theta q \delta h<0 \quad$ and $\quad q\left(1-\frac{2 s^{*}}{K}\right) \pi^{2}-\psi h y^{*}<0$.

\section{Appendix A.4. Predator-free equilibrium}

If $y^{*}=0$, we have

$$
r\left(1-\frac{x^{*}}{k}\right) x^{*}=0, \quad q\left(1-\frac{s^{*}}{K}\right) s^{*}=0
$$

so the possible predator-free equilibria are

$$
\left(x^{*}, s^{*}, y^{*}\right) \in\{(0,0,0),(0, K, 0),(k, 0,0),(k, K, 0)\},
$$

and these are all feasible. The equilibrium $(0,0,0)$ was found to be unstable in Section Appendix A.1. At $(0, K, 0)$, the Jacobian matrix is

$$
J=\left[\begin{array}{ccc}
r & 0 & 0 \\
0 & -q & -\frac{\psi K}{\pi} \\
0 & 0 & \frac{\eta \psi K}{\pi}-\delta
\end{array}\right]
$$

The Jacobian has at least one positive eigenvalue, namely $\lambda_{1}=$ $r$, therefore $(0, K, 0)$ is an unstable equilibrium point. Similarly, the equilibrium point $(k, 0,0)$ is unstable, since the Jacobian matrix would have positive eigenvalue $q$. At $(k, K, 0)$, the Jacobian is

$$
J=\left[\begin{array}{ccc}
-r & 0 & -\theta k / \pi \\
0 & -q & -\psi K / \pi \\
0 & 0 & (\epsilon \theta k+\eta \psi K) / \pi-\delta
\end{array}\right]
$$

with eigenvalues $-r,-q$ and $(\epsilon \theta k+\eta \psi K) / \pi-\delta$, so the stability condition is

$$
\frac{\epsilon \theta k+\eta \psi K}{\pi}-\delta<0
$$

\section{Appendix A.5. Positive equilibrium}

Conditions for the feasibility of the positive equilibrium for the model (41)-(43) were previously discussed in the appendix of Mougi (2010), with numerical simulations supporting some of their assertions. We shall now go through the feasibility and stability conditions for this state in detail.

We now examine the feasibility of the positive steady state, $x^{*}, s^{*}, y^{*}>0$. From (A.2), we have

$$
y^{*}=q\left(1-\frac{s^{*}}{K}\right)\left(x^{*}+s^{*}+h\right),
$$

so for $y^{*}>0$ we require $s^{*}<K$. Using (A.3), we obtain $x^{*}$ in terms of $s^{*}$ :

$$
x^{*}=\frac{\delta-\eta \psi}{\epsilon \theta-\delta} s^{*}+\frac{\delta h}{\epsilon \theta-\delta} .
$$


Thus, for $x^{*}>0$, we require

$$
\frac{\delta-\eta \psi}{\epsilon \theta-\delta} s^{*}+\frac{\delta h}{\epsilon \theta-\delta}>0
$$

Substituting (A.1) into (A.3), an alternative expression for $x^{*}$ is found:

$$
x^{*}=k-\frac{k \theta q}{\psi r}\left(1-\frac{s^{*}}{k}\right)
$$

Equating the two expressions for $x^{*}$ and rearranging for $s^{*}$, we arrive at

$$
s^{*}=\frac{k\left(1-\frac{\theta q}{\psi r}\right)-\frac{\delta h}{\epsilon \theta-\delta}}{\frac{\delta-\eta \psi}{\epsilon \theta-\delta}-\frac{k \theta q}{\psi r K}} .
$$

Combining the feasibility conditions for $x^{*}$ and $y^{*}$ and imposing $s^{*}>0$, we must have

$$
\begin{cases}0<\frac{k\left(1-\frac{\theta q}{\psi r}\right)-\frac{\delta h}{\epsilon \theta-\delta}}{\frac{\delta \eta \eta \psi}{\epsilon \theta-\delta}-\frac{k \theta q}{\psi r K}}<\min \left\{K, \frac{\delta h}{\eta \psi-\delta}\right\}, & \text { if } \frac{\eta \psi-\delta}{\epsilon \theta-\delta}>0, \\ \frac{k\left(1-\frac{\theta q}{\psi r}\right)-\frac{\delta \phi}{\epsilon \theta-\delta}}{\frac{\delta-\eta \psi}{\epsilon \theta-\delta}-\frac{k \theta q}{\psi r K}}>\max \left\{0, \frac{\delta h}{\eta \psi-\delta}\right\}, & \text { if } \frac{\eta \psi-\delta}{\epsilon \theta-\delta}<0 .\end{cases}
$$

We are able to carry out stability analysis of the positive equilibrium, similar to that of Deka et al. (2016). The Jacobian is given by (A.4). A necessary condition for the positive equilibrium to be stable is that the characteristic polynomial of $J$, $\omega(\lambda)=\lambda^{3}+\omega_{1} \lambda^{2}+\omega_{2} \lambda+\omega_{3}$, has positive coefficients. These coefficients are

$$
\begin{gathered}
\omega_{1}=-\operatorname{tr}(J), \\
\omega_{2}=J_{11} J_{22}-\left(J_{12} J_{21}+J_{13} J_{31}+J_{23} J_{32}\right), \\
\omega_{3}=-\operatorname{det}(J) .
\end{gathered}
$$

By the Routh-Hurwitz criteria (Gantmakher, 1998), a necessary and sufficient condition for these coefficients to be positive is

$\omega_{1}>0, \quad \operatorname{det}\left[\begin{array}{ccc}\omega_{1} & 1 & 0 \\ \omega_{3} & \omega_{2} & \omega_{1} \\ 0 & 0 & \omega_{3}\end{array}\right]>0$, and $\operatorname{det}\left[\begin{array}{cc}\omega_{1} & 1 \\ \omega_{3} & \omega_{2}\end{array}\right]>0$

Therefore, the positive steady state is locally stable if and only if

$$
\omega_{1}, \omega_{3}>0 \text { and } \omega_{1} \omega_{2}>\omega_{3},
$$

where

$$
\begin{gathered}
\omega_{1}=-\left(H_{1}^{*}+H_{2}^{*}\right), \\
\omega_{2}=H_{1}^{*} H_{2}^{*}-\frac{\theta \psi}{\pi^{4}} x^{*} s^{*} y^{* 2}+\frac{\theta x^{*} y^{*}}{\pi^{3}}\left(\epsilon \theta\left(s^{*}+h\right)-\eta \psi s^{*}\right) \\
+\frac{\psi s^{*} y^{*}}{\pi^{3}}\left(\eta \psi\left(x^{*}+h\right)-\epsilon \theta x^{*}\right), \\
\omega_{3}=\left(\frac{\psi}{\pi^{3}} H_{1}^{*} s^{*} y^{*}-\frac{\theta \psi}{\pi^{5}} x^{*} s^{*} y^{* 2}\right)\left(\eta \psi\left(x^{*}+h\right)-\epsilon \theta x^{*}\right) \\
+\left(\frac{\theta}{\pi^{3}} H_{2}^{*} x^{*} y^{*}-\frac{\theta \psi}{\pi^{5}} x^{*} s^{*} y^{* 2}\right)\left(\epsilon \theta\left(s^{*}+h\right)-\eta \psi s^{*}\right), \\
H_{1}^{*}=r\left(1-\frac{2 x^{*}}{k}\right)-\frac{\theta}{\pi^{2}}\left(s^{*}+h\right) y^{*}, \\
H_{2}^{*}=q\left(1-\frac{2 s^{*}}{K}\right)-\frac{\psi}{\pi^{2}}\left(x^{*}+h\right) y^{*} .
\end{gathered}
$$

\section{References}

Abrams, P. A., 2006. The effects of switching behavior on the evolutionary diversification of generalist consumers. The American Naturalist 168 (5), 645-659.

Alonso, D., Bartumeus, F., Catalan, J., 2002. Mutual interference between predators can give rise to turing spatial patterns. Ecology 83 (1), 28-34.

Amarasekare, P., 2007. Spatial dynamics of communities with intraguild predation: the role of dispersal strategies. The American Naturalist 170 (6), 819-831.

Andersen, M., 1991. Properties of some density-dependent integrodifference equation population models. Mathematical Biosciences 104 (1), 135-157.

Anderson, R., 2005. A hybrid mathematical model of solid tumour invasion: the importance of cell adhesion. Mathematical Medicine and Biology: a Journal of the IMA 22 (2), 163-186.

Armsworth, P. R., Roughgarden, J. E., 2005. The impact of directed versus random movement on population dynamics and biodiversity patterns. The American Naturalist 165 (4), 449-465.

Bacaër, N., 2011. Verhulst and the logistic equation (1838). In: A Short History of Mathematical Population Dynamics. Springer, pp. 35-39.

Baggio, J. A., Salau, K., Janssen, M. A., Schoon, M. L., Bodin, Ö., 2011. Landscape connectivity and predator-prey population dynamics. Landscape Ecology 26 (1), 33-45.

Barnes Jr, V. G., 1990. The influence of salmon availability on movements and range of brown bears on southwest Kodiak Island. Bears: Their Biology and Management, 305-313.

Bassett, A., Krause, A. L., Van Gorder, R. A., 2017. Continuous dispersal in a model of predator-prey-subsidy population dynamics. Ecological Modelling 354, 115-122.

Baurmann, M., Gross, T., Feudel, U., 2007. Instabilities in spatially extended predator-prey systems: spatio-temporal patterns in the neighborhood of turing-hopf bifurcations. Journal of Theoretical Biology 245 (2), 220-229.

Berns, V. D., Atwell, G. C., Boone, D. L., 1980. Brown bear movements and habitat use at Karluk Lake, Kodiak Island. Bears: Their Biology and Management, 293-296.

Berns, V. D., Hensel, R. J., 1972. Radio tracking brown bears on kodiak island. Bears: Their Biology and Management, 19-25.

Briggs, C. J., Hoopes, M. F., 2004. Stabilizing effects in spatial parasitoid-host and predator-prey models: a review. Theoretical population biology 65 (3), 299-315.

Burkey, T. V., 1989. Extinction in nature reserves: the effect of fragmentation and the importance of migration between reserve fragments. Oikos, 75-81.

Cantrell, R. S., Cosner, C., 2004. Spatial ecology via reaction-diffusion equations. John Wiley \& Sons.

Chen, X., Hambrock, R., Lou, Y., 2008. Evolution of conditional dispersal: a reaction-diffusion-advection model. Journal of mathematical biology 57 (3), 361-386.

Council, N. R., 1996. Rock fractures and fluid flow: contemporary understanding and applications. National Academies Press.

Crowder, L., Norse, E., 2008. Essential ecological insights for marine ecosystem-based management and marine spatial planning. Marine policy 32 (5), 772-778

Crowley, P. H., 1981. Dispersal and the stability of predator-prey interactions. The American Naturalist 118 (5), 673-701.

Deka, B., Patra, A., Tushar, J., Dubey, B., 2016. Stability and hopf-bifurcation in a general gauss type two-prey and one-predator system. Applied Mathematical Modelling 40 (11-12), 5793-5818.

El Abdllaoui, A., Auger, P., Kooi, B. W., De la Parra, R. B., Mchich, R., 2007. Effects of density-dependent migrations on stability of a two-patch predatorprey model. Mathematical Biosciences 210 (1), 335-354.

Gable, T. D., Windels, S. K., Bruggink, J. G., Homkes, A. T., 2016. Where and how wolves (Canis lupus) kill beavers (Castor canadensis). PLoS One 11 (12), e0165537.

Gable, T. D., Windels, S. K., Romanski, M. C., Rosell, F., 2018. The forgotten prey of an iconic predator: a review of interactions between grey wolves canis lupus and beavers castor spp. Mammal Review 48 (2), 123-138.

Gamito, S., 1998. Growth models and their use in ecological modelling: an application to a fish population. Ecological Modelling 113 (1-3), 83-94.

Gantmakher, F. R., 1998. The theory of matrices. Vol. 131. American Mathematical Soc.

Genkai-Kato, M., Yamamura, N., 1999. Unpalatable prey resolves the paradox 
of enrichment. Proceedings of the Royal Society of London B: Biological Sciences 266 (1425), 1215-1219.

Gogan, P., Route, W., Olexa, E., Thomas, N., Kuehn, D., Podruzny, K., 2004. Gray wolves in and adjacent to Voyageurs National Park, Minnesota: Research and synthesis 1987-1991. Tech. rep., National Park Service.

Goldwyn, E. E., Hastings, A., 2008. When can dispersal synchronize populations? Theoretical population biology 73 (3), 395-402.

Goldwyn, E. E., Hastings, A., 2009. Small heterogeneity has large effects on synchronization of ecological oscillators. Bulletin of mathematical biology 71 (1), 130-144.

Grindrod, P., 1988. Models of individual aggregation or clustering in single and multi-species communities. Journal of Mathematical Biology 26 (6), 651660

Guo, Q., Taper, M., Schoenberger, M., Brandle, J., 2005. Spatial-temporal population dynamics across species range: from centre to margin. Oikos 108 (1), 47-57.

Gurney, W. S., Veitch, A. R., 2000. Self-organization, scale and stability in a spatial predator-prey interaction. Bulletin of Mathematical Biology 62 (1), 61-86.

Hamilton, C. D., Kovacs, K. M., Ims, R. A., Aars, J., Lydersen, C., 2017. An arctic predator-prey system in flux: climate change impacts on coastal space use by polar bears and ringed seals. Journal of Animal Ecology.

Hanski, I., 1998. Metapopulation dynamics. Nature 396 (6706), 41

Hassell, M. P., Comins, H. N., Mayt, R. M., 1991. Spatial structure and chaos in insect population dynamics. Nature 353 (6341), 255.

Hauzy, C., Gauduchon, M., Hulot, F. D., Loreau, M., 2010. Density-dependent dispersal and relative dispersal affect the stability of predator-prey metacommunities. Journal of Theoretical Biology 266 (3), 458-469.

Hauzy, C., Hulot, F. D., Gins, A., Loreau, M., 2007. Intra-and interspecific density-dependent dispersal in an aquatic prey-predator system. Journal of Animal Ecology 76 (3), 552-558.

Holling, C. S., 1959. The components of predation as revealed by a study of small-mammal predation of the european pine sawfly1. The Canadian Entomologist 91 (5), 293-320.

Holmes, E. E., Lewis, M. A., Banks, J., Veit, R., 1994. Partial differential equations in ecology: spatial interactions and population dynamics. Ecology 75 (1), 17-29.

Holt, R. D., 1984. Spatial heterogeneity, indirect interactions, and the coexistence of prey species. The American Naturalist 124 (3), 377-406.

Hopper, K. R., Roush, R. T., 1993. Mate finding, dispersal, number released, and the success of biological control introductions. Ecological entomology 18 (4), 321-331.

Huang, Y., Diekmann, O., 2001. Predator migration in response to prey density: what are the consequences? Journal of mathematical biology 43 (6), 561581.

Hutson, V., Vickers, G., 1983. A criterion for permanent coexistence of species, with an application to a two-prey one-predator system. Mathematical Biosciences 63 (2), 253-269.

Jansen, J. E., Van Gorder, R. A., 2018. Dynamics from a predator-prey-quarryresource-scavenger model. Theoretical Ecology 11, 19-38.

Jansen, V. A., 1995. Regulation of predator-prey systems through spatial interactions: a possible solution to the paradox of enrichment. Oikos, 384-390.

Jansen, V. A., 2001. The dynamics of two diffusively coupled predator-prey populations. Theoretical Population Biology 59 (2), 119-131.

Jansen, V. A., de Roos, A. M., et al., 2000. The role of space in reducing predator-prey cycles. The geometry of ecological interactions: simplifying spatial complexity, 183-201.

Jansen, V. A., Lloyd, A. L., 2000. Local stability analysis of spatially homogeneous solutions of multi-patch systems. Journal of mathematical biology 41 (3), 232-252.

Johnston, C. A., 2017. Beavers: Boreal Ecosystem Engineers. Springer

Kang, Y., Sasmal, S. K., Messan, K., 2015. A two-patch prey-predator model with dispersal in predators driven by the strength of predation. arXiv preprint arXiv: 1505.03820

Kareiva, P., 1990. Population dynamics in spatially complex environments: theory and data. Philosophical Transactions: Biological Sciences 330 (1257), 175-190.

Kurowski, L., Krause, A. L., Mizuguchi, H., Grindrod, P., Van Gorder, R. A., 2017. Two-species migration and clustering in two-dimensional domains. Bulletin of mathematical biology 79 (10), 2302-2333.

Levin, S., 2003. Complex adaptive systems: exploring the known, the unknown and the unknowable. Bulletin of the American Mathematical Society 40 (1), $3-19$.

Levin, S. A., 1974. Dispersion and population interactions. The American Naturalist 108 (960), 207-228

Levin, S. A., 1976. Population dynamic models in heterogeneous environments. Annual Review of Ecology and Systematics 7 (1), 287-310.

Levy, D., Harrington, H. A., Van Gorder, R. A., 2016. Role of seasonality on predator-prey-subsidy population dynamics. Journal of theoretical biology 396, 163-181.

Lewis, M. A., Maini, P. K., Petrovskii, S. V., 2013. Dispersal, individual movement and spatial ecology. Lecture Notes in Mathematics (Mathematics Bioscience Series) 2071.

Liebhold, A., Koenig, W. D., Bjørnstad, O. N., 2004. Spatial synchrony in population dynamics. Annu. Rev. Ecol. Evol. Syst. 35, 467-490.

Lima, S. L., 2002. Putting predators back into behavioral predator-prey interactions. Trends in Ecology \& Evolution 17 (2), 70-75.

Maciel, G. A., Kraenkel, R. A., 2014. How population loss through habitat boundaries determines the dynamics of a predator-prey system. Ecological complexity 20, 33-42.

Malthus, T. R., Appleman, P., 1976. An essay on the principle of population: Text, sources and background. Criticism.

Metz, H., 2000. The geometry of ecological interactions: simplifying spatial complexity. Cambridge University Press.

Moinfar, A., Varavei, A., Sepehrnoori, K., Johns, R. T., 2013. Development of a coupled dual continuum and discrete fracture model for the simulation of unconventional reservoirs. In: SPE Reservoir Simulation Symposium. Society of Petroleum Engineers.

Mougi, A., 2010. Coevolution in a one predator-two prey system. PloS one 5 (11), e13887.

Murray, J. D., 2002. Mathematical Biology. I, Volume 17 of Interdisciplinary Applied Mathematics.

Nadell, C. D., Drescher, K., Foster, K. R., 2016. Spatial structure, cooperation and competition in biofilms. Nature Reviews Microbiology 14 (9), 589.

Nathan, R., 2001. The challenges of studying dispersal. Trends in Ecology \& Evolution 16 (9), 481-483.

Neubert, M. G., Klepac, P., Van den Driessche, P., 2002. Stabilizing dispersal delays in predator-prey metapopulation models. Theoretical Population Biology 61 (3), 339-347.

Nevai, A. L., Van Gorder, R. A., 2012. Effect of resource subsidies on predatorprey population dynamics: a mathematical model. Journal of Biological Dynamics 6 (2), 891-922.

Okubo, A., Levin, S. A., 2013. Diffusion and ecological problems: modern perspectives. Vol. 14. Springer Science \& Business Media.

Okubo, A., Maini, P., Williamson, M., Murray, J., 1989. On the spatial spread of the grey squirrel in britain. Proceedings of the Royal Society of London B: Biological Sciences 238 (1291), 113-125

Pascual, M., 1993. Diffusion-induced chaos in a spatial predator-prey system. Proc. R. Soc. Lond. B 251 (1330), 1-7.

Petrovskii, S., Li, B.-L., Malchow, H., 2004. Transition to spatiotemporal chaos can resolve the paradox of enrichment. Ecological Complexity 1 (1), 37-47.

Pilyugin, S. S., Medlock, J., De Leenheer, P., 2016. The effectiveness of marine protected areas for predator and prey with varying mobility. Theoretical population biology $110,63-77$.

Plitzko, S. J., Drossel, B., 2015. The effect of dispersal between patches on the stability of large trophic food webs. Theoretical Ecology 8 (2), 233-244.

Ranta, E., Kaitala, V., Lundberg, P., 1997. The spatial dimension in population fluctuations. Science 278 (5343), 1621-1623.

Reimchen, T., 2000. Some ecological and evolutionary aspects of bear-salmon interactions in coastal british columbia. Canadian Journal of Zoology 78 (3), 448-457.

Ripa, J., 2000. Analysing the moran effect and dispersal: their significance and interaction in synchronous population dynamics. Oikos 89 (1), 175-187.

Roemer, G. W., Donlan, C. J., Courchamp, F., 2002. Golden eagles, feral pigs, and insular carnivores: How exotic species turn native predators into prey. Proceedings of the National Academy of Sciences 99 (2), 791-796.

Rosenzweig, M. L., 1971. Paradox of enrichment: destabilization of exploitation ecosystems in ecological time. Science 171 (3969), 385-387.

Rosenzweig, M. L., MacArthur, R. H., 1963. Graphical representation and stability conditions of predator-prey interactions. The American Naturalist 97 (895), 209-223.

Roy, S., Chattopadhyay, J., 2007. The stability of ecosystems: a brief overview 
of the paradox of enrichment. Journal of Biosciences 32 (2), 421-428.

Ruokolainen, L., Abrams, P. A., McCann, K. S., Shuter, B. J., 2011. The roles of spatial heterogeneity and adaptive movement in stabilizing (or destabilizing) simple metacommunities. Journal of theoretical biology 291, 76-87.

Scheffer, M., De Boer, R. J., 1995. Implications of spatial heterogeneity for the paradox of enrichment. Ecology 76 (7), 2270-2277.

Shen, L., Van Gorder, R. A., 2017. Predator-prey-subsidy population dynamics on stepping-stone domains. Journal of Theoretical Biology 420, 241-258.

Shiga, T., 1988. Stepping stone models in population genetics and population dynamics. In: Stochastic Processes in Physics and Engineering. Springer, pp. 345-355.

Suzuki, K., Yoshida, T., 2012. Non-random spatial coupling induces desynchronization, chaos and multistability in a predator-prey-resource system. Journal of theoretical biology 300, 81-90.

ter Braak, C. J., Hanski, I., Verboom, J., 1998. The incidence function approach to modeling of metapopulation dynamics. Modeling spatiotemporal dynamics in ecology, 167-188.

Tilman, D., Kareiva, P. M., 1997. Spatial ecology: the role of space in population dynamics and interspecific interactions. Vol. 30. Princeton University Press.

Toyokawa, W., 2017. Scrounging by foragers can resolve the paradox of enrichment. Royal Society open science 4 (3), 160830.

Van Kirk, R. W., Lewis, M. A., 1997. Integrodifference models for persistence in fragmented habitats. Bulletin of Mathematical Biology 59 (1), 107.

Volterra, V., 1928. Variations and fluctuations of the number of individuals in animal species living together. ICES Journal of Marine Science 3 (1), 3-51.

Weisser, W. W., Hassell, M. P., 1996. Animals' on the move'stabilize hostparasitoid systems. Proceedings of the Royal Society of London B: Biological Sciences 263 (1371), 749-754.

Weisser, W. W., Jansen, V. A., Hassell, M. P., 1997. The effects of a pool of dispersers on host-parasitoid systems. Journal of Theoretical Biology 189 (4), 413-425.

Williamson, M., 1996. Biological invasions. Vol. 15. Springer Science \& Business Media.

Willson, M. F., 1993. Mammals as seed-dispersal mutualists in north america. Oikos, 159-176.

Windels, S. K., 2017. Beavers as engineers of wildlife habitat. In: Beavers: Boreal Ecosystem Engineers. Springer, pp. 239-268.

Yamauchi, A., Yamamura, N., 2005. Effects of defense evolution and diet choice on population dynamics in a one-predator-two-prey system. Ecology 86 (9), 2513-2524. 